\title{
Distribution and population status of rare plant species in the Marmarosh Mountains (Ukrainian Carpathians)
}

\author{
Yuriy KOBIV ${ }^{1,2}$, Andriy PROKOPIV², Viktor NACHYCHKO², Liubov BORSUKEVYCH², Myroslava HELESH ${ }^{2}$ \\ ${ }^{1}$ Institute of Ecology of the Carpathians, National Academy of Sciences of Ukraine \\ 4, Kozelnytska Str., Lviv 79026, Ukraine \\ ykobiv@gmail.com \\ ${ }^{2}$ Botanical Garden of Ivan Franko National University of Lviv \\ 44, Cheremshyny Str., Lviv 79014, Ukraine \\ botsad@franko.lviv.ua
}

Kobiv Y., Prokopiv A., Nachychko V., Borsukevych L., Helesh M. Distribution and population status of rare plant species in the Marmarosh Mountains (Ukrainian Carpathians). Ukr. Bot. J., 2017, 74(2): 163-176.

Abstract. The article presents the results of multiple surveys of habitats of rare plant species in the Marmarosh Mts, Ukrainian Carpathians, and depicts their exact location and population parameters. Along with the analysis of earlier literature and herbarium data, this enabled to reveal some spatio-temporal patterns in their distribution, namely habitat characteristics and trends in population dynamics, which allowed to determine their conservation status. Numerous facts of decline and extinction, as well as examples of positive dynamics in populations of the studied species were described. Cold-dependent species (Cerastium cerastoides, Primula minima, Veronica bellidioides) proved to be vulnerable to climate change particularly at the lower limit of their distribution. Another factor of impact is decrease of grazing, which triggers vegetation succession and consequent replacement of some low-competitive rare plants (Genista tinctoria subsp. oligosperma, Koeleria macrantha subsp. transsilvanica) with shrubs or tussock graminoids. However, another group of rare species (Heracleum sphondylium subsp. transsilvanicum, Gentiana punctata, Jovibarba globifera subsp. preissiana, Veronica fruticans) benefits from the decrease of anthropogenic impact.

Keywords: climate change, extinction, population dynamics, locality, Carpathians

\section{Introduction}

The Marmarosh (or Maramureş - in Romanian transcription) Mountains are situated on the UkrainianRomanian border and belong to the Inner Carpathians. They have a very diverse geological structure with prevailing crystalline bedrock and rather frequent limestone outcrops. This significantly differs from the vast outer part of the Ukrainian Carpathians dominated by mostly uniform acidic sandstone flysch (Kondracki, 1989). The Marmarosh Mts are the steepest in the Ukrainian Carpathians (Kruhlov, 2008), therefore rock outcrops are quite common here. That mountain range is the second highest (after the Chornohora) massif in the Ukrainian Carpathians with two summits of Mt. Pip Ivan exceeding $1900 \mathrm{~m}$ a.s.l. This territory makes an isolated alpine "environmental island". In the Marmarosh Mts some rare and endemic plants are confined to the eastern edge of their ranges and do not occur elsewhere in the Ukrainian Carpathians. All the above reasons make these mountains unique in terms of their biodiversity and landscape value, therefore their considerable part was included into the Carpathian

CYu. KOBIV, A. PROKOPIV, V. NACHYCHKO, L. BORSUKEVYCH, M. HELESH, 2017

Укр. бот. журн., 2017, 74(2)
Biosphere Reserve. The flora and vegetation of the Marmarosh Mts have been studied since the late $19^{\text {th }}$ century. The most precise old chorological data concerning distribution of rare species were published by H. Zapałowicz (1889), I. Klášterský (1929-1931), V. Krist (1935), and M. Deyl (1940). Floristic studies during the Soviet period, i.e. in the 1950-1980s, were rather incomplete because of the limited access to that frontier territory, which remained one of the most understudied regions of the Ukrainian Carpathians. Though the "Flora of the Ukrainian SSR" (Flora RSS Ucr., 1950-1965), as well as V.I. Chopyk's (1976) and K.A. Malynovski's (1980) monographs contain much information on the flora of the Marmarosh Mts, most publications from the last decades lack the exact data on the localities of rare species and the status of their populations in the region.

The aim of this study is to fill that gap, i.e. to specify their distribution in the Marmarosh Mts and compare contemporary data with those contained in literature and herbaria in order to reveal possible dynamic patterns. Special attention was paid to population parameters of the rare species, their viability and conservation status. We indicated the exact location of their habitats to set a baseline for future monitoring. 


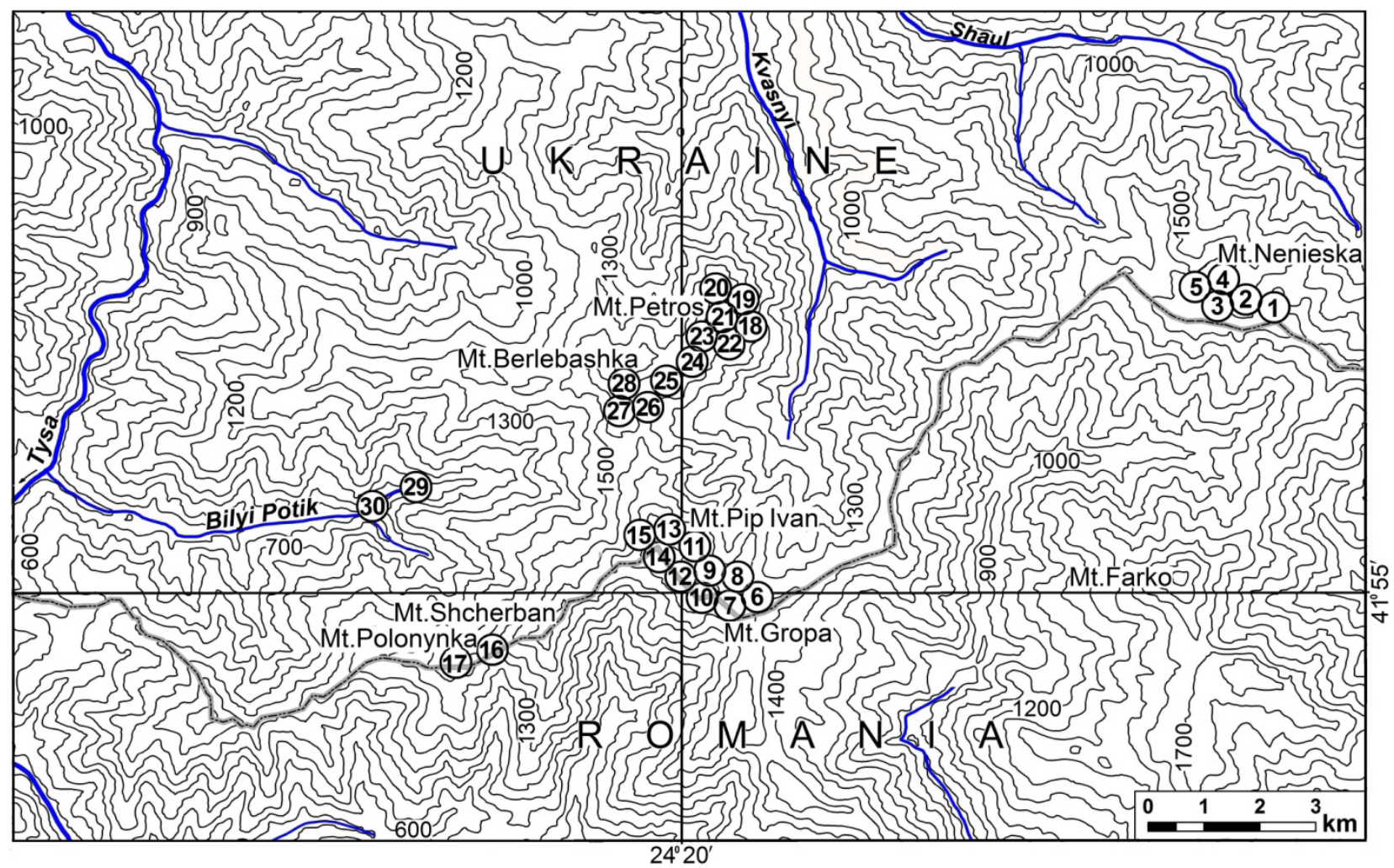

Studied localities of rare species in the Marmarosh Mts. 1, 2 ... 30 - locality numbers

Although the presented data on the rare species are not fully comprehensive, they are supposed to depict properly their distribution and population status in the target region.

\section{Materials and Methods}

The results presented below were obtained during field surveys carried out in 2002, 2006, 2010, and 2016 in the Marmarosh Mts, Ukrainian Carpathians. The studied area stretches about $15 \mathrm{~km}$ along the UkrainianRomanian border (Figure).

Thorough analysis of the previous publications and herbarium data on rare species had been performed before surveying their known localities in situ.

The map of the target area with locations of the surveyed habitats of rare species is provided in the Figure where their numeration corresponds to Tables 1-3.

The species were considered as rare following K.A. Malynovski et al. (2002) compendium.

Density of populations was estimated on sets of square plots placed randomly using $0.5 \times 0.5 \mathrm{~m}^{2}$ or $0.1 \times$ $0.1 \mathrm{~m}^{2}$ frames with 10-fold repetition (Harper, 1977).
In small populations their total census was estimated (Elzinga et al., 2009).

Species abundance in Tables 1, 2 is presented according to J. Braun-Blanquet (1964) scale.

Exact site locations were determined in WGS-84 system with Garmin eTrex GPS navigator with the accuracy of measurements within 10 metres.

Content of calcium and magnesium in soil was measured complexometrically and $\mathrm{pH}\left(\mathrm{H}_{2} \mathrm{O}\right)$ value electrometrically (Arinushkina, 1970).

Nomenclature of vascular plants follows "Flora Europaea" (1964-1980), with modern updates for endemics by J. Kliment et al. (2016), and M.O. Hill et al. (2006) - for bryophytes.

\section{Results and Discussion}

The habitats of rare species are mostly confined to sites with special ecological conditions, geological structure or landforms, which can be considered as "hot spots" of their occurrence (Ziman et al., 2009). For instance, in the Ukrainian Carpathians those are usually alpine, rocky, marshy or calcareous localities (Kobiv, 2010). 
Therefore, our results are presented with regard to such hotspots.

Mt. Nenieska. This site is remarkable for its ca. $500 \mathrm{~m}$ long steep rocky north-eastern slope with calciterich sandstone bedrock. It is a habitat of many rare saxicolous species, most of which are calciphilous (Table 1). One of them, Leontopodium alpinum (L.) Cass., we discovered in two localities $(\# 3,4)$, where only about a dozen of its flowering individuals occur. Local rangers confirm that the population number has decreased significantly during the last decades, because the plants have been illegally dug up by souvenir hunters. That has undermined the population viability and only some solitary individuals have survived in the most inaccessible rocky loci. Therefore, the species is critically endangered.

The rocks of Mt. Nenieska harbor an EastCarpathian endemic Astragalus australis (L.) Lam. subsp. krajinae (Domin) Domin, which is restricted to steep slopes (localities 2, 3) where the density of its flowering individuals is about 0.3 specimens per $\mathrm{m}^{2}$. The population is maintained by seed recruitment.

Another rare saxicolous species, Aster alpinus L., is abundant in the same localities. Its clones with numerous flowering shoots may cover up to $200 \mathrm{~cm}^{2}$.

The rocks are inhabited by a highly viable population of Primula halleri J.F. Gmel. The average density of its flowering individuals is 0.05 specimens per $\mathrm{m}^{2}$. The only way for recruitment of this short-lived species is seed reproduction.

Other noteworthy alpine rare taxa are: Cerastium alpinum L. subsp. lanatum (Lam.) Asch. \& Graebn., Erigeron alpinus L., Minuartia verna (L.) Hiern. subsp. gerardii (Willd.) Graebn., and Ranunculus thora L., which occur here on a rather large area of about 5 hectares though they do not exhibit high density (localities 2-5). Scarce patches of sward vegetation harbor Achillea lingulata Waldst. \& Kit.

Steep shady and moist rocks provide habitats for a rare East-South-Carpathian endemic Achillea oxyloba (DC.) Sch. Bip. subsp. schurii (Sch. Bip.) Heimerl. Due to clonal growth it forms dense patches up to 200-300 $\mathrm{cm}^{2}$ with numerous flowering shoots (localities 2, 5).

Much more abundant are Hieracium villosum Jacq. which forms dense clones on rocky shelves and Botrychium lunaria (L.) Sw. mostly confined to loci of almost barren gravel. Rhodiola rosea $\mathrm{L}$. that occupies both types of these habitats is also rather frequent but subjected to severe damage due to illegal extirpation of its rhizomes for medicinal purposes.
A small population of an East-South-Carpathian endemic, Heracleum sphondylium L. subsp. transsilvanicum (Schur) Brummitt $(=H$. palmatum Baumg.), is situated in a steep hollow near the summit of Mt. Nenieska. Its core zone is confined to the tallforb community, which covers about $30 \mathrm{~m}^{2}$ (locality 1). Density of flowering undividuals of $H$. sphondylium subsp. transsilvanicum is 1.8 specimens per $1 \mathrm{~m}^{2}$. This aclonal species is capable only of seed reproduction.

All the mentioned species are restricted to the subalpine zone within 1700-1800 m a.s.l. In terms of occurrence of the rare plants (for instance, Achillea oxyloba subsp. schurii, Allium montanum, Aster alpinus, Astragalus australis subsp. krajinae, Draba siliquosa, Erigeron alpinus, Leontopodium alpinum, Primula halleri), the rocks of Mt. Nenieska have most resemblance to saxicolous sites from the Svydovets Mts that apparently can be explained by similar geological structure.

The next cluster of rare species' localities is the highest part of the Marmarosh Mts, i.e. Mt. Pip Ivan with adjacent Mt. Gropa, Mt. Shcherban (aka Zherban), and Mt. Polonynka built mostly of crystalline bedrock - gneisses, metamorphic schists and amphibolites. Considerable area is covered with alpine vegetation there. Snowbed habitats that harbor highly specialized prostrate cold-adapted species are most characteristic of the alpine environment. One of such chionophilous species is Salix herbacea L. We found its two small populations (localities 10,12) confined to snowbeds on the highest elevations at the ridge on Mt. Pip Ivan on poorly insolated N and NW aspects. Due to clonal growth $S$. herbacea forms dense mats, while its patches cover small areas of 15 and $4 \mathrm{~m}^{2}$.

Another chionophilous species is Cerastium cerastoides (L.) Britton, which inhabits bottoms of large snowbeds in the glacial cirques of Mt. Pip Ivan. It occurs mostly on gravel sites with poor floristic composition and low abundance of other herbaceous plants (locality 8). Cerastium cerastoides can form mats up to 100$150 \mathrm{~cm}^{2}$ with $15-20$ flowering shoots. However, flowering is not common and most individuals are vegetative and stunted. The species is rare now and we found only two its small populations restricted to 1745$1820 \mathrm{~m}$ a.s.l. altitudinal range. It is remarkable that according to M. Deyl (1940) it was much more abundant in the late 1930s being recorded in 19 localities within 1600-1910 m a.s.l. Moreover, earlier H. Zapałowicz (1889) had reported on its still lower locality at 1580 $\mathrm{m}$ a.s.l. Apparently, decline of $C$. cerastoides is caused 
Table 1. Floristic composition in localities of rare plant species in the hotspots of Mt. Nenieska and Mt. Pip Ivan with adjacent area (names of rare species in boldface)

\begin{tabular}{|c|c|c|c|c|c|c|c|c|c|c|c|c|c|c|c|c|c|}
\hline Number of locality & 1 & 2 & 3 & 4 & 5 & 6 & 7 & 8 & 9 & 10 & 11 & 12 & 13 & 14 & 15 & 16 & 17 \\
\hline Location & \multicolumn{5}{|c|}{ Mt. Nenieska } & \multicolumn{2}{|c|}{$\begin{array}{c}\text { Mt. } \\
\text { Gropa }\end{array}$} & \multicolumn{8}{|c|}{ Mt. Pip Ivan } & \multicolumn{2}{|c|}{$\begin{array}{c}\text { Mt. } \\
\text { Polonynka }\end{array}$} \\
\hline Date of relevé (day, month, year) & $\begin{array}{l}\stackrel{8}{\circ} \\
\grave{0} \\
\dot{\vec{\lambda}}\end{array}$ & 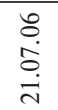 & 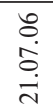 & 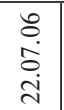 & 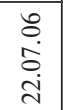 & 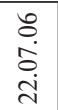 & $\begin{array}{l}\text { o } \\
\stackrel{0}{0} \\
\text { ì }\end{array}$ & $\begin{array}{l}0 \\
\stackrel{0}{0} \\
0 \\
\ddot{\imath}\end{array}$ & 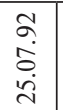 & 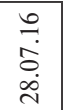 & $\begin{array}{l}\text { ô. } \\
\hat{0} \\
\text { ஸे }\end{array}$ & $\begin{array}{l}0 \\
\stackrel{0}{0} \\
0 \\
\stackrel{0}{0}\end{array}$ & 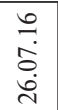 & 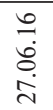 & 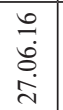 & 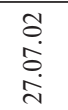 & 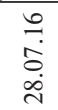 \\
\hline Area of relevé $\left(\mathrm{m}^{2}\right)$ & 25 & 100 & 25 & 25 & 25 & 100 & 100 & 25 & 100 & 4 & 100 & 15 & 25 & 10 & 15 & 25 & 2 \\
\hline Elevation (m a.s.l.) & $\stackrel{2}{2}$ & 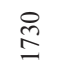 & $\stackrel{\infty}{=}$ & $\stackrel{n}{\cong}$ & $\stackrel{ }{n}$ & ஜn & $\stackrel{\mathscr{0}}{\infty}$ & $\stackrel{ }{n}$ & $\tilde{\infty}$ & $\begin{array}{l}n \\
\infty \\
\infty\end{array}$ & $\stackrel{\circ}{\infty}$ & $\cong$ & $\stackrel{2}{\cong}$ & $\stackrel{ }{2}$ & 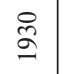 & $\stackrel{n}{n}$ & $\stackrel{n}{\tilde{o}}$ \\
\hline Exposition & NE & NE & $\mathrm{NE}$ & $\mathrm{NE}$ & $\mathrm{NE}$ & $\mathrm{E}$ & $\mathrm{E}$ & NE & NE & NW & NE & $\mathrm{N}$ & $\mathrm{NE}$ & $\mathrm{E}$ & $\mathrm{NE}$ & NW & $\mathrm{NE}$ \\
\hline Inclination $\left(^{\circ}\right)$ & 40 & 45 & 50 & 50 & 50 & 2 & 20 & 15 & 30 & 40 & 20 & 30 & 15 & 50 & 15 & 3 & 70 \\
\hline Vegetation cover $(\%)$ & 100 & 70 & 40 & 70 & 40 & 90 & 90 & 50 & 50 & 60 & 80 & 70 & 59 & 50 & 70 & 100 & 25 \\
\hline Achillea lingulata & $\cdot$ & $\cdot$ & + & $\cdot$ & $\cdot$ & $\cdot$ & + & $\cdot$ & $\cdot$ & $\cdot$ & $\cdot$ & $\cdot$ & $\cdot$ & $\cdot$ & $\cdot$ & $\cdot$ & $\cdot$ \\
\hline Achillea oxyloba subsp. schurii & $\cdot$ & + & $\cdot$ & $\cdot$ & + & $\cdot$ & $\cdot$ & $\cdot$ & $\cdot$ & $\cdot$ & $\cdot$ & . & $\cdot$ & $\cdot$ & $\cdot$ & $\cdot$ & $\cdot$ \\
\hline Achillea strictag & $\cdot$ & $\cdot$ & + & $\cdot$ & $\cdot$ & $\cdot$ & + & $\cdot$ & $\cdot$ & $\cdot$ & + & $\cdot$ & $\cdot$ & + & $\cdot$ & $\cdot$ & $\cdot$ \\
\hline Allium montanum & . & + & . & $\cdot$ & $\cdot$ & . & $\cdot$ & $\cdot$ & $\cdot$ & $\cdot$ & . & . & . & $\cdot$ & $\cdot$ & $\cdot$ & $\cdot$ \\
\hline Alnus viridis & . & + & $\cdot$ & . & 1 & . & $\cdot$ & . & $\cdot$ & . & . & . & . & $\cdot$ & . & . & . \\
\hline Anemone narcissifolia & $\cdot$ & $\cdot$ & $\cdot$ & $\cdot$ & $\cdot$ & $\cdot$ & + & $\cdot$ & $\cdot$ & $\cdot$ & $\cdot$ & $\cdot$ & $\cdot$ & $\cdot$ & + & $\cdot$ & $\cdot$ \\
\hline Anthemis cretica subsp. pyrethriformis & $\cdot$ & $\cdot$ & $\cdot$ & $\cdot$ & $\cdot$ & $\cdot$ & + & $\cdot$ & $\cdot$ & $\cdot$ & $\cdot$ & $\cdot$ & $\cdot$ & + & + & $\cdot$ & . \\
\hline Anthoxanthum alpinum & $\cdot$ & . & + & . & $\cdot$ & + & + & . & + & . & . & 2 & . & $\cdot$ & + & . &. \\
\hline Arnica montana & . & $\cdot$ & $\cdot$ & . & $\cdot$ & $\cdot$ & + & $\cdot$ & $\cdot$ & . & . & $\cdot$ & $\cdot$ & $\cdot$ & + & . & $\cdot$ \\
\hline Aster alpinus & . & 1 & + & $\cdot$ & $\cdot$ & $\cdot$ & $\cdot$ & $\cdot$ & $\cdot$ & . & $\cdot$ & $\cdot$ & $\cdot$ & . & $\cdot$ & . & $\cdot$ \\
\hline Astragalus australis subsp. krajinae & $\cdot$ & + & + & $\cdot$ & $\cdot$ & $\cdot$ &. &. & $\cdot$ & $\cdot$ & $\cdot$ &. &. & $\cdot$ & $\cdot$ & $\cdot$ &. \\
\hline Botrychium lunaria & $\cdot$ & $\cdot$ & $\cdot$ & + & $\cdot$ & $\cdot$ & . & $\cdot$ & $\cdot$ & . & $\cdot$ & $\cdot$ & . & . & $\cdot$ & $\cdot$ & $\cdot$ \\
\hline Calamagrostis villosa & 1 & 2 & $\cdot$ & $\cdot$ & $\cdot$ & $\cdot$ & 2 & $\cdot$ & $\cdot$ & $\cdot$ & . & $\cdot$ & . & $\cdot$ & $\cdot$ & 2 & . \\
\hline Campanula alpina & $\cdot$ & $\cdot$ & $\cdot$ & $\cdot$ & $\cdot$ & $\cdot$ & . & $\cdot$ & + & 1 & $\cdot$ & $\cdot$ & . & $\cdot$ & + & . & . \\
\hline Campanula carpatica & $\cdot$ & $\cdot$ & . & $\cdot$ & $\cdot$ & $\cdot$ & $\cdot$ & $\cdot$ & $\cdot$ & $\cdot$ & . & $\cdot$ & $\cdot$ & $\cdot$ & . & $\cdot$ & 2 \\
\hline Campanula kladniana & $\cdot$ & $\cdot$ & + &. & $\cdot$ & $\cdot$ & $\cdot$ & $\cdot$ & + & $\cdot$ & + & $\cdot$ & + & 1 & + & $\cdot$ &. \\
\hline Cardaminopsis halleri & $\cdot$ & $\cdot$ & $\cdot$ & $\cdot$ & + & $\cdot$ & $\cdot$ & $\cdot$ & $\cdot$ & $\cdot$ & $\cdot$ & $\cdot$ & $\cdot$ & $\cdot$ & $\cdot$ & $\cdot$ & $\cdot$ \\
\hline Carduus kerneri & $\cdot$ & + & $\cdot$ & $\cdot$ & $\cdot$ & $\cdot$ & . & $\cdot$ & $\cdot$ & $\cdot$ & $\cdot$ & $\cdot$ & . & + & $\cdot$ & . & $\cdot$ \\
\hline Carex limosa & . & $\cdot$ & $\cdot$ & . & $\cdot$ & 2 & $\cdot$ & $\cdot$ & $\cdot$ & . & . & . & . & $\cdot$ & $\cdot$ & . & $\cdot$ \\
\hline Carex pauciflora & $\cdot$ & $\cdot$ & $\cdot$ & $\cdot$ & $\cdot$ & 2 & $\cdot$ & $\cdot$ & $\cdot$ & $\cdot$ & $\cdot$ &. &. & $\cdot$ & $\cdot$ & $\cdot$ & $\cdot$ \\
\hline Carex sempervirens & $\cdot$ & 1 & $\cdot$ & $\cdot$ & 1 & . & 4 & $\cdot$ & 2 & 1 & 2 & $\cdot$ & $\cdot$ & 3 & 3 & $\cdot$ & $\cdot$ \\
\hline Cerastium cerastoides & $\cdot$ & $\cdot$ & . & $\cdot$ & $\cdot$ & $\cdot$ & . & + & . & . & . & . & . & . & . & $\cdot$ & . \\
\hline Cerastium lanatum & $\cdot$ & + & + & + & $\cdot$ & $\cdot$ & $\cdot$ & $\cdot$ & $\cdot$ & $\cdot$ &. & $\cdot$ & $\cdot$ & $\cdot$ & $\cdot$ & $\cdot$ & $\cdot$ \\
\hline Cirsium erisithales & $\cdot$ & + & + & + & $\cdot$ & $\cdot$ & $\cdot$ & $\cdot$ & $\cdot$ & $\cdot$ & $\cdot$ & $\cdot$ & $\cdot$ & $\cdot$ & $\cdot$ & $\cdot$ & $\cdot$ \\
\hline Deschampsia caespitosa & $\cdot$ & $\cdot$ & $\cdot$ & $\cdot$ & $\cdot$ & + & $\cdot$ & 3 & 1 & $\cdot$ & 1 & + &. & $\cdot$ & $\cdot$ & 1 & $\cdot$ \\
\hline Draba siliquosa & $\cdot$ & $\cdot$ & + & $\cdot$ & $\cdot$ & $\cdot$ & . & $\cdot$ & $\cdot$ & $\cdot$ & $\cdot$ & $\cdot$ & $\cdot$ & $\cdot$ & $\cdot$ & $\cdot$ & $\cdot$ \\
\hline Erigeron alpinus & . & $\cdot$ & + & + & . & . & . & . & $\cdot$ & . & . & . & . & $\cdot$ & . & . & $\cdot$ \\
\hline Festuca airoides & $\cdot$ & . & 1 & 4 & $\cdot$ & $\cdot$ & $\cdot$ & $\cdot$ & $\cdot$ & 2 & . & $\cdot$ & $\cdot$ & $\cdot$ & . & $\cdot$ & 3 \\
\hline Festuca carpatica & $\cdot$ & $\cdot$ & + & $\cdot$ & $\cdot$ & $\cdot$ & $\cdot$ & $\cdot$ & $\cdot$ & . & . & $\cdot$ & $\cdot$ & $\cdot$ & . & . & . \\
\hline Festuca picta & $\cdot$ & + & 1 & 1 & $\cdot$ & $\cdot$ & 2 & + & 1 & $\cdot$ & $\cdot$ & 1 & $\cdot$ & $\cdot$ & 2 & $\cdot$ & $\cdot$ \\
\hline Festuca rubra & $\cdot$ & $\cdot$ & 2 & $\cdot$ & . & $\cdot$ & . & $\cdot$ & . & $\cdot$ & . & $\cdot$ & . & $\cdot$ & . & 4 & $\cdot$ \\
\hline Galium anisophyllon & $\cdot$ & $\cdot$ & + & + & $\cdot$ & $\cdot$ & $\cdot$ & $\cdot$ & $\cdot$ & $\cdot$ & . & $\cdot$ & . & + & $\cdot$ & $\cdot$ & $\cdot$ \\
\hline Galium erectum subsp. suberectum & . & + & + & $\cdot$ & . & $\cdot$ & . & . & $\cdot$ & . & . & . & . & $\cdot$ & . & . & . \\
\hline Gentiana acaulis & $\cdot$ & $\cdot$ & $\cdot$ & + & $\cdot$ & $\cdot$ & $\cdot$ & $\cdot$ & + &. & $\cdot$ & $\cdot$ &. &. & + & $\cdot$ & $\cdot$ \\
\hline Gentiana asclepiadea & $\cdot$ & + & $\cdot$ & $\cdot$ & $\cdot$ & $\cdot$ & . & $\cdot$ & $\cdot$ &. & + & . & . & $\cdot$ & $\cdot$ & $\cdot$ &. \\
\hline Gentiana lutea & $\cdot$ & $\cdot$ & $\cdot$ & . & $\cdot$ & $\cdot$ & . & $\cdot$ & $\cdot$ & $\cdot$ & 1 & $\cdot$ & . & . & . & . & $\cdot$ \\
\hline Gentiana punctata & $\cdot$ & $\cdot$ & $\cdot$ & $\cdot$ & $\cdot$ & $\cdot$ & $\cdot$ & $\cdot$ & $\cdot$ & $\cdot$ & + & + & $\cdot$ & $\cdot$ &. &. & $\cdot$ \\
\hline Heracleum palmatum & 2 & $\cdot$ & $\cdot$ & $\cdot$ & $\cdot$ & $\cdot$ & . & $\cdot$ & $\cdot$ & $\cdot$ & $\cdot$ & $\cdot$ & . & $\cdot$ & $\cdot$ & . & $\cdot$ \\
\hline Hieracium alpinum & . & $\cdot$ & . & $\cdot$ & $\cdot$ & $\cdot$ & $\cdot$ & $\cdot$ & $\cdot$ & + & $\cdot$ & + & $\cdot$ & $\cdot$ & . & $\cdot$ & $\cdot$ \\
\hline Hieracium umbellatum & $\cdot$ & $\cdot$ & $\cdot$ & $\cdot$ & $\cdot$ & $\cdot$ & . & . & $\cdot$ & $\cdot$ & . & $\cdot$ & . & + & $\cdot$ & $\cdot$ & 1 \\
\hline Hieracium villosum & $\cdot$ & + & $\cdot$ & + & . & $\cdot$ & $\cdot$ & . & $\cdot$ & $\cdot$ & $\cdot$ & $\cdot$ & $\cdot$ & $\cdot$ & $\cdot$ & $\cdot$ & $\cdot$ \\
\hline Homogyne alpina & . & $\cdot$ & $\cdot$ & $\cdot$ & $\cdot$ & + & + & $\cdot$ & $\cdot$ & + & $\cdot$ & $\cdot$ & $\cdot$ & $\cdot$ & $\cdot$ & 1 & $\cdot$ \\
\hline Huperzia selago & . & $\cdot$ & $\cdot$ & $\cdot$ & $\cdot$ & $\cdot$ & $\cdot$ & $\cdot$ & $\cdot$ & + & $\cdot$ & + & . & $\cdot$ & $\cdot$ & $\cdot$ & $\cdot$ \\
\hline Hypericum richeri subsp. grisebachii & $\cdot$ & + & $\cdot$ & . & $\cdot$ & $\cdot$ & $\cdot$ & $\cdot$ & $\cdot$ & $\cdot$ & + & + & $\cdot$ & 1 & + & + & $\cdot$ \\
\hline Hypochoeris uniflora & $\cdot$ & $\cdot$ & $\cdot$ & $\cdot$ & $\cdot$ & $\cdot$ & . & . & $\cdot$ & $\cdot$ & $\cdot$ & $\cdot$ & $\cdot$ & + & + & $\cdot$ & + \\
\hline Juncus trifidus & $\cdot$ & $\cdot$ & $\cdot$ & $\cdot$ & $\cdot$ & $\cdot$ & $\cdot$ & $\cdot$ & 2 & 2 & $\cdot$ & $\cdot$ & 3 & 1 & $\cdot$ & $\cdot$ & $\cdot$ \\
\hline Juniperus communis subsp. alpina & . & . & $\cdot$ & . & . & . & . & . & 2 & . & . & . & . & . & + & . & 2 \\
\hline Knautia dipsacifolia & . & + & + & $\cdot$ & $\cdot$ & $\cdot$ & . & . & $\cdot$ & $\cdot$ & $\cdot$ & $\cdot$ & . & $\cdot$ & $\cdot$ & $\cdot$ & . \\
\hline Laserpitum alpinum & . & $\cdot$ & + & $\cdot$ & $\cdot$ & $\cdot$ & $\cdot$ & . & + & . & + & $\cdot$ & . & + & $\cdot$ & + & 1 \\
\hline
\end{tabular}




\begin{tabular}{|c|c|c|c|c|c|c|c|c|c|c|c|c|c|c|c|c|c|}
\hline Number of locality & 1 & 2 & 3 & 4 & 5 & 6 & 7 & 8 & 9 & 10 & 11 & 12 & 13 & 14 & 15 & 16 & 17 \\
\hline Leontopodium alpinum & $\cdot$ & $\cdot$ & + & + & $\cdot$ & $\cdot$ & $\cdot$ & $\cdot$ & $\cdot$ & $\cdot$ & $\cdot$ & $\cdot$ & $\cdot$ & $\cdot$ & $\cdot$ & $\cdot$ & $\cdot$ \\
\hline Leucanthemum vulgare subsp. alpicola & $\cdot$ & $\cdot$ & $\cdot$ & $\cdot$ & . & $\cdot$ & $\cdot$ & . & + & . & $\cdot$ & . & $\cdot$ & $\cdot$ & + & $\cdot$ & . \\
\hline Linum extraaxillare & $\cdot$ & $\cdot$ & + & + & . & . & $\cdot$ & $\cdot$ & $\cdot$ & . & $\cdot$ & . & . & $\cdot$ & $\cdot$ & . & . \\
\hline Lotus corniculatus & . & . & 1 & + & . & . & . & . & . & . & . & . & $\cdot$ & + & + & . & . \\
\hline Luzula luzuloides & $\cdot$ & 2 & 2 & 1 & 1 & $\cdot$ & + & $\cdot$ & 2 & $\cdot$ & 3 & . & . & $\cdot$ & $\cdot$ & $\cdot$ & $\cdot$ \\
\hline Ligusticum mutellina & $\cdot$ & . & $\cdot$ & . & $\cdot$ & + & + & $\cdot$ & $\cdot$ & $\cdot$ & + & 2 & 2 & . & $\cdot$ & . & $\cdot$ \\
\hline Minuaria verna subsp. gerardii & . & $\cdot$ & + & + & $\cdot$ & $\cdot$ & $\cdot$ & $\cdot$ & $\cdot$ & $\cdot$ & $\cdot$ & $\cdot$ & . & . & $\cdot$ & $\cdot$ & $\cdot$ \\
\hline Narcissus angustifolius & . & $\cdot$ & $\cdot$ & $\cdot$ & $\cdot$ & $\cdot$ & . & $\cdot$ & $\cdot$ & . & . & $\cdot$ & . & . & $\cdot$ & + & . \\
\hline Nardus stricta & . &. & . & . & . & + & . & + & . & . & . & . & . & . & . & . & . \\
\hline Pedicularis hacquetii & $\cdot$ & + & $\cdot$ & $\cdot$ & + & $\cdot$ & $\cdot$ & $\cdot$ & $\cdot$ & $\cdot$ & $\cdot$ & $\cdot$ & $\cdot$ & $\cdot$ & $\cdot$ & $\cdot$ & $\cdot$ \\
\hline Phyteuma orbiculare & . & + & + & . & $\cdot$ & $\cdot$ & $\cdot$ & $\cdot$ & $\cdot$ & $\cdot$ & . & . & . & . & $\cdot$ & . & . \\
\hline Phyteuma vagneri & $\cdot$ & $\cdot$ & $\cdot$ & $\cdot$ & $\cdot$ & $\cdot$ & + & $\cdot$ & + & $\cdot$ & + & $\cdot$ & $\cdot$ & + & + & + & $\cdot$ \\
\hline Poa granitica subsp. disparilis & $\cdot$ & $\cdot$ & + & $\cdot$ & . & $\cdot$ & $\cdot$ & 2 & $\cdot$ & . & $\cdot$ & 1 & 3 & $\cdot$ & + & $\cdot$ & . \\
\hline Polygala amara subsp. brachyptera & $\cdot$ & $\cdot$ & $\cdot$ & + & $\cdot$ & $\cdot$ & $\cdot$ & $\cdot$ & $\cdot$ & $\cdot$ & . & . & . & $\cdot$ & $\cdot$ & $\cdot$ & $\cdot$ \\
\hline Polygonum bistorta & $\cdot$ & $\cdot$ & $\cdot$ & $\cdot$ & $\cdot$ & + & $\cdot$ & $\cdot$ & $\cdot$ & $\cdot$ & + & $\cdot$ & . & $\cdot$ & $\cdot$ & + & $\cdot$ \\
\hline Potentilla aurea & $\cdot$ & $\cdot$ & + & $\cdot$ & $\cdot$ & $\cdot$ & + & $\cdot$ & $\cdot$ & $\cdot$ & $\cdot$ & . & + & $\cdot$ & + & $\cdot$ & . \\
\hline Potentilla crantzii & . & $\cdot$ & $\cdot$ & $\cdot$ & . & $\cdot$ & $\cdot$ & $\cdot$ & . & . & . & . & . & 1 & $\cdot$ & . & . \\
\hline Primula elatior & . & + & . & + & + & . & . & . & . & . & . & . & . &. & . & . & . \\
\hline Primula halleri & $\cdot$ & + & + & + & $\cdot$ & $\cdot$ & $\cdot$ & $\cdot$ & . & . & $\cdot$ & . & . & . & $\cdot$ & $\cdot$ & . \\
\hline Primula minima & $\cdot$ & $\cdot$ & $\cdot$ & $\cdot$ & $\cdot$ & $\cdot$ & $\cdot$ & $\cdot$ & $\cdot$ & + & $\cdot$ & . & $\cdot$ & $\cdot$ & $\cdot$ & . & . \\
\hline Pulsatilla alba & $\cdot$ & $\cdot$ & $\cdot$ & $\cdot$ & $\cdot$ & $\cdot$ & 1 & $\cdot$ & + & 1 & $\cdot$ & $\cdot$ & . & + & + & $\cdot$ & $\cdot$ \\
\hline Ranunculus thora & $\cdot$ & + & + & $\cdot$ & + & $\cdot$ & $\cdot$ & $\cdot$ & $\cdot$ & $\cdot$ & $\cdot$ & . & $\cdot$ & $\cdot$ & $\cdot$ & . & $\cdot$ \\
\hline Rhodiola rosea & $\cdot$ & + & . & + & . & $\cdot$ & . & . & $\cdot$ & $\cdot$ & . & . & $\cdot$ & $\cdot$ & . & . & $\cdot$ \\
\hline Rhododendron myrtifolium & $\cdot$ & $\cdot$ & $\cdot$ & $\cdot$ & $\cdot$ & $\cdot$ & 1 & $\cdot$ & 1 & 4 & $\cdot$ & $\cdot$ & $\cdot$ & $\cdot$ & + & $\cdot$ & $\cdot$ \\
\hline Rumex carpaticus & + & $\cdot$ & $\cdot$ & $\cdot$ & $\cdot$ & $\cdot$ & $\cdot$ & $\cdot$ & $\cdot$ & $\cdot$ & + & $\cdot$ & $\cdot$ & $\cdot$ & $\cdot$ & $\cdot$ & . \\
\hline Salix herbacea & $\cdot$ & . & . & $\cdot$ & . & . & . & . & . & 2 & $\cdot$ & 4 & . & . & $\cdot$ & . & . \\
\hline Saxifraga paniculata & . & . & . & + & 1 & . & . & . & . & . & . & . & . & . & . & . & . \\
\hline Scabiosa lucida subsp. barbata & . & + & + & $\cdot$ & . & $\cdot$ & $\cdot$ & $\cdot$ & . & $\cdot$ & $\cdot$ & . & $\cdot$ & 1 & + & $\cdot$ & . \\
\hline Scorzonera rosea & . & $\cdot$ & $\cdot$ & $\cdot$ & $\cdot$ & $\cdot$ & + & $\cdot$ & $\cdot$ & $\cdot$ & + & . & . & $\cdot$ & $\cdot$ & + & $\cdot$ \\
\hline Sedum alpestre & . & + & + & $\cdot$ & $\cdot$ & $\cdot$ & $\cdot$ & $\cdot$ & $\cdot$ & . & $\cdot$ & . & 4 & + & + & $\cdot$ & + \\
\hline Sempervivum carpathicum subsp. carpathicum & . & $\cdot$ & $\cdot$ & $\cdot$ & $\cdot$ & $\cdot$ & $\cdot$ & $\cdot$ & + & $\cdot$ & . & . & . & $\cdot$ & + & $\cdot$ & . \\
\hline Silene dubia & $\cdot$ & + & + & $\cdot$ & $\cdot$ & $\cdot$ & $\cdot$ & $\cdot$ & $\cdot$ & $\cdot$ & $\cdot$ & $\cdot$ & $\cdot$ & $\cdot$ & $\cdot$ & $\cdot$ & $\cdot$ \\
\hline Silene vulgaris & $\cdot$ & + & + & + & $\cdot$ & $\cdot$ & $\cdot$ & $\cdot$ & $\cdot$ & $\cdot$ & $\cdot$ & $\cdot$ & $\cdot$ & $\cdot$ & + & $\cdot$ & $\cdot$ \\
\hline Soldanella hungarica & . & $\cdot$ & $\cdot$ & $\cdot$ & . & + & $\cdot$ & $\cdot$ & $\cdot$ & $\cdot$ & $\cdot$ & 2 & . & . & $\cdot$ & . & . \\
\hline Solidago virgaurea subsp. minuta & $\cdot$ & $\cdot$ & $\cdot$ & $\cdot$ & . & $\cdot$ & . & . & + & . & + & + & . & . & + & $\cdot$ & $\cdot$ \\
\hline Thymus alpestris & $\cdot$ & + & + & + & $\cdot$ & $\cdot$ & $\cdot$ & $\cdot$ & $\cdot$ & $\cdot$ & + & $\cdot$ & $\cdot$ & + & + & $\cdot$ & 1 \\
\hline Vaccinium myrtillus & . & $\cdot$ & + & $\cdot$ & . & + & . & . & 1 & . & . & + & 2 & 1 & + & . & . \\
\hline Vaccinium vitis-idaea & . & $\cdot$ & . & . & $\cdot$ & $\cdot$ & $\cdot$ & $\cdot$ & $\cdot$ & 1 & $\cdot$ & . & . & . & + & $\cdot$ & . \\
\hline Valeriana tripteris & + & + & $\cdot$ & $\cdot$ & + & $\cdot$ & $\cdot$ & $\cdot$ & $\cdot$ & . & $\cdot$ & . & . & . & $\cdot$ & $\cdot$ & . \\
\hline Veronica baumgartenii & $\cdot$ & $\cdot$ & $\cdot$ & $\cdot$ & + & $\cdot$ & $\cdot$ & $\cdot$ & $\cdot$ & + & $\cdot$ & . & . & $\cdot$ & $\cdot$ & $\cdot$ & . \\
\hline Veronica bellidioides & $\cdot$ & $\cdot$ & $\cdot$ & . & $\cdot$ & $\cdot$ & $\cdot$ & . & $\cdot$ & $\cdot$ & $\cdot$ & $\cdot$ & $\cdot$ & $\cdot$ & + & $\cdot$ & $\cdot$ \\
\hline \multicolumn{18}{|l|}{ BRYOPHYTES } \\
\hline Polytrichum sexangulare & $\cdot$ & $\cdot$ & $\cdot$ & $\cdot$ & $\cdot$ & $\cdot$ & $\cdot$ & 3 & $\cdot$ & 1 & $\cdot$ & $\cdot$ & $\cdot$ & $\cdot$ & $\cdot$ & $\cdot$ & $\cdot$ \\
\hline Racomitrium heterostichum & . & $\cdot$ & . & . & . & $\cdot$ & . & 4 & . & 3 & . & . & . & . & $\cdot$ & $\cdot$ & . \\
\hline Sphagnum russowii & . & . & . & . & . & . & . & . & . & 2 & . & 3 & . & . & . & . & . \\
\hline Tortella tortuosa & $\cdot$ & 2 & $\cdot$ & 2 & $\cdot$ & $\cdot$ & $\cdot$ & $\cdot$ & $\cdot$ & . & . & . & 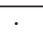 & . & . &. & 3 \\
\hline
\end{tabular}

Other species occurring in a single locality (number in brackets) - Adenostyles alliariae (1): 2; Alchemilla sp. (5): +; Asplenium septentrionale (17): +; Caltha laeta (6): +; Campanula serrata (17): +; Cardaminopsis halleri (5): +; Carex atrata (2): +; C. canescens (6): 1; C. echinata (6): 2; C. nigra (6): 1; C. ornithopoda (17): +; C. rostrata (6): 3; Carlina acaulis (4): +; Centaurea mollis (2): +; Cicerbita alpina (1): 1; Cirsium waldsteinii (1): 2; Clematis alpina (2): +; Dianthus compactus (7): +; Eriophorum polystachyon (6): 3; E. vaginatum (6): +; Geranium sylvaticum subsp. alpestre (1): +; Gymnadenia conopsea (15): +; Leontodon croceus (15): +; Lilium martagon (2): +; Luzula alpino-pilosa (12): +; L. sudetica (6): +; Molinia caerulea (6): 2; Parnassia palustris (3): +; Picea abies (16): 2; Polygonum viviparum (5): +; Pulmonaria filarszkyana (2): +; Ranunculus carpaticus (1): +; Sedum telephium subsp. fabaria (14): +; Senecio nemorensis (1): +; Symphytum cordatum (2): +; Thesium alpinum (14): +; Vaccinium gaultherioides (15): +; Veratrum lobelianum (12): +; Viola biflora (5): +; V. declinata (7): +; Cetraria islandica (10): 1; Dicranum scoparium (10): 3; Grimmia anodon (17): 1; Homalothecium philippeanum (17): 2; Hylocomium splendens (10): 3; Lophozia excisa (13): 4; Pohlia nutans (13): 3; Polytrichastrum alpinum (12): 3; Pseudoleskeella nervosa (17): 1; Sphagnum sp. (6): 4. Location of the sites: $1-47^{\circ} 57^{\prime} 44,9^{\prime \prime} \mathrm{N} 24^{\circ} 27^{\prime} 53,0^{\prime \prime} \mathrm{E} ; 2-47^{\circ} 57^{\prime} 54,2^{\prime \prime} \mathrm{N} 24^{\circ} 27^{\prime} 21,6^{\prime \prime} \mathrm{E} ; 3-47^{\circ} 57^{\prime} 56,9^{\prime \prime} \mathrm{N} 24^{\circ} 27^{\prime} 18,6^{\prime \prime} \mathrm{E} ; 4-$ $47^{\circ} 57^{\prime} 59,1^{\prime \prime} \mathrm{N} 24^{\circ} 27^{\prime} 17,0^{\prime \prime} \mathrm{E} ; 5-47^{\circ} 58^{\prime} 07,0^{\prime \prime} \mathrm{N} 24^{\circ} 27^{\prime} 09,7^{\prime \prime} \mathrm{E} ; 6-47^{\circ} 54^{\prime} 58,2^{\prime \prime} \mathrm{N} 24^{\circ} 20^{\prime} 58,0^{\prime \prime} \mathrm{E} ; 7-47^{\circ} 55^{\prime} 05,4^{\prime \prime} \mathrm{N} 24^{\circ} 20^{\prime} 20,6^{\prime \prime} \mathrm{E}$; $8-47^{\circ} 55^{\prime} 19,3^{\prime \prime} \mathrm{N} 24^{\circ} 19^{\prime} 48,7^{\prime \prime} \mathrm{E} ; 9-47^{\circ} 55^{\prime} 39,5^{\prime \prime} \mathrm{N} 24^{\circ} 16^{\prime} 41,0^{\prime \prime} \mathrm{E} ; 10-47^{\circ} 55^{\prime} 15,8^{\prime \prime} \mathrm{N} 24^{\circ} 19^{\prime} 48,6^{\prime \prime} \mathrm{E} ; 11-47^{\circ} 55^{\prime} 83,0^{\prime \prime} \mathrm{N}$ $24^{\circ} 19^{\prime} 38,9^{\prime \prime} \mathrm{E} ; 12$ - $47^{\circ} 55^{\prime} 27,6^{\prime \prime} \mathrm{N} 24^{\circ} 19^{\prime} 37,4^{\prime \prime} \mathrm{E} ; 13-47^{\circ} 55^{\prime} 35,4^{\prime \prime} \mathrm{N} 24^{\circ} 19^{\prime} 38,0^{\prime \prime} \mathrm{E} ; 14-47^{\circ} 55^{\prime} 33,0^{\prime \prime} \mathrm{N} 24^{\circ} 19^{\prime} 31,4^{\prime \prime} \mathrm{E} ; 15-$ $47^{\circ} 55^{\prime} 35,2^{\prime \prime} \mathrm{N} 24^{\circ} 19^{\prime} 30,5^{\prime \prime} \mathrm{E} ; 16-47^{\circ} 54^{\prime} 27,2^{\prime \prime} \mathrm{N} 24^{\circ} 17^{\prime} 01,1^{\prime \prime} \mathrm{E} ; 17-47^{\circ} 54^{\prime} 20,6^{\prime \prime} \mathrm{N} 24^{\circ} 16^{\prime} 39,5^{\prime \prime} \mathrm{E}$. 
mainly by shrinkage of its snowbed habitats due to decreasing thickness and duration of snowpack that has been described in the Carpathians recently (Micu, 2009). Interestingly, M. Deyl (1940) admitted that some largest snowbeds on Mt. Pip Ivan even persisted throughout some growth seasons in the 1930s, though that has never happened in the last decades.

The aforementioned chionophylous species are adapted to long-lasting snow cover, short growth period and are confined to snowbed communities that belong to the class Salicetea herbaceae Br.-B1. 1948. They are low-competitive and most viable on moss-dominated or barren patches with initial soil. As a result of warminginduced succession they are being gradually replaced by more competitive sward-forming cold-tolerant graminoids, for instance Poa granitica Br.-Bl. subsp. disparilis (Nyár.) Nyár. (=P. deylii Chrtek \& V. Jirásek). The latter taxon, which is endemic to the Eastern and Southern Carpathians, is rather common in the alpine zone of Mt. Pip Ivan.

Primula minima L. is also a cold-adapted lowstatured species, which forms patches of 25-40 rosettes per $100 \mathrm{~cm}^{2}$. It occurs at saxicolous alpine habitats up to the highest elevations, while the lowest locality that we found on Mt. Shcherban is on $1750 \mathrm{~m}$ a.s.l. Interestingly, M. Deyl (1940) reported the species locality situated $100 \mathrm{~m}$ lower, while H. Zapałowicz (1889) - as low as on $1580 \mathrm{~m}$ a.s.l.

A frequent component of many alpine communities is Sedum alpestre Vill. It is noteworthy that in one of the glacial cirques of Mt. Pip Ivan (locality 13) this species dominates in the snowbed community and forms dense mats that cover up to $0.25 \mathrm{~m}^{2}$, which is uncommon for other regions of the Carpathians.

It is remarkable that in population replenishment of all the aforementioned alpine cold-tolerant species vegetative reproduction markedly prevails over seed recruitment. That provides for their better persistence in harsh climatic conditions.

One of the rarest and most endangered species, Veronica bellidioides L., occurs on the ridge at the NW summit of Mt. Pip Ivan, which is its only locality still remaining in the Ukrainian Carpathians (Kobiv, 2009). The area of its saxicolous habitat is merely about $15 \mathrm{~m}^{2}$ (locality 15). Monitoring of the population showed that the number of flowering individuals has decreased in 14 years from 25 to only 3 specimens by 2016, which is critical, because seed recruitment is crucial for further survival of the species. Moreover, the density of its seedlings has decreased dramatically. Analysis of the past data (Klášterský, 1931; Deyl, 1940) shows that the species was much more abundant there in the 1930s, and the lowermost limit of its distribution has shifted about $100 \mathrm{~m}$ upwards in the altitudinal terms since then. This proves that currently $V$. bellidioides is at the brink of extinction in Ukraine. Such dynamics is in line with the decline of the species in the Sudetes (Szczęśniak et al., 2011) at comparatively low elevations, as well as upward shifts of its altitudinal limits in the Alps (Pauli et al., 2007; Frei et al., 2010), which apparently have resulted from climate change.

Another cold-adapted species, Luzula spicata L., has also demonstrated vulnerability to climate change and consequent decline in the Alps (Pauli et al., 2007; Frei et al., 2010). It was reported from the very top of Mt. Pip Ivan at the end of the $19^{\text {th }}$ century by $\mathrm{H}$. Zapałowicz (1889), but we failed to find it in the Marmarosh Mts. Most probably, L. spicata, which was documented in several highest massifs of the Ukrainian Carpathians in the late $19^{\text {th }}-$ early $20^{\text {th }}$ century, has become completely extinct in the region by now, because it has not been confirmed anywhere in Ukraine since World War II.

We also did not manage to reconfirm the occurrence of another chionophylous species, Saxifraga carpatica Sternb., which is documented by an old herbarium specimen from Mt. Pip Ivan ( LWS 46140).

According to the European compendia on ecological indicator values (Ellenberg et al., 1992; Zarzycki et al., 2002), the above mentioned species, which show declining trends in their distribution in the Marmarosh Mts (Cerastium cerastoides, Luzula spicata, Primula minima, Saxifraga carpatica, Veronica bellidioides), belong to the most cold-adapted plants in the Carpathian flora. Their Marmarosh habitats are restricted to the lowermost limits of their ecological ranges and the ongoing climate change has an adverse impact on them.

We managed to find the locality of an arctic-alpine saxicolous species Potentilla crantzii (Crantz) Beck ex Fritsch (locality 14) on a steep rocky slope at the NW summit of Mt. Pip Ivan, which was reported by M. Deyl (1940) but has not been reconfirmed ever since. The area of the site is only about $10 \mathrm{~m}^{2}$. The species produces compact clones with numerous subprostrate flowering shoots.

An East-South-Carpathian endemic Anthemis cretica L. subsp. pyrethriformis (Schur) Govaerts (= A. carpatica Waldst. \& Kit. ex Willd. subsp. pyrethriformis (Schur) Prodan) is restricted to the high-mountain zone of Mts Gropa, Pip Ivan and Shcherban. This is 
the only area in Ukraine where it occurs. Apparently, A. cretica subsp. pyrethriformis forms a metapopulation comprised of a number of local populations scatthered within a rather narrow stripe along the UkrainianRomanian border. This suffruticose plant occurs on rocky and grassland habitats mostly in the alpine zone. Its highest density (up to 20 flowering individuals per $\mathrm{m}^{2}$ ) was noted alongside of the tourist path (locality 7) in the gaps among the heath or herbaceous vegetation, which result from moderate trampling. Such gaps provide suitable microhabitats for seed recruitment of $A$. cretica subsp. pyrethriformis (Kobiv, 2012). Its lowermost locality is situated at $1520 \mathrm{~m}$ a.s.l. at the SW foot of Mt. Shcherban, where the density of individuals has decreased by 1.8 times over the 14 -year monitoring period.

Rhododendron myrtifolium Schott \& Kotschy, a narrow-range high-mountain species, has a similar distribution pattern. It is very abundant in the massif of Mt. Pip Ivan and occurs up to the highest elevations (localities 10, 15).

A large metapopulation of a pan-Carpathian endemic Sempervivum carpathicum Wettst. ex Prodan subsp. carpathicum ( $=S$. montanum L. subsp. carpathicum (Wettst. ex Prodan) Wettst. ex A. Berg.) inhabits rocky outcrops in the high-mountain zone of Mt. Pip Ivan within about 5 -hectare area. The species produces mats of numerous rosettes that may cover up to $300 \mathrm{~cm}^{2}$. The population is sustained primarily by vegetative reproduction provided by stolon growth.

Gentiana lutea L., a high-mountain Central European species, is widely spread in glacial cirques on NE slopes of Mt. Pip Ivan and Mt. Gropa within 1600-1900 m a.s.l. The species occurs either in the open grassland habitats or at the edge of scrub vegetation. The largest population on Mt. Pip Ivan (locality 11) covers ca. 4 hectares and includes 3.5-5.5 thousand flowering individuals. Density of flowering shoots in clusters may reach 4 specimens per $\mathrm{m}^{2}$.

On the saddle between Mt. Shcherban and Mt. Polonynka (locality 16) we found Narcissus angustifolius Curtis, a species protected by the Bern Convention. Its population, which numbers ca. 100 flowering individuals occupies about $30 \mathrm{~m}^{2}$.

Not far from that site, on the rocks at the W summit of Mt. Polonynka we found a small locality of Campanula carpatica Jacq., a pan-Carpathian endemic (locality 17). It occurs on an area of only about $2 \mathrm{~m}^{2}$, apparently being a fragment of the large metapopulation situated mostly on the Romanian side. The species forms lax clones with about 1.5 flowering stems per $100 \mathrm{~cm}^{2}$.

Gentiana punctata L., a Central European alpine species, is very common in the Marmarosh Mts. Due to clonal growth it may form clumps up to $3 \mathrm{~m}^{2}$ with $8-10$ flowering shoots per $1 \mathrm{~m}^{2}$.

As follows from the above data, the massif of Mt. Pip Ivan with the adjacent territory is a remarkable hotspot, where localities of many rare alpine species are concentrated on a comparatively small area (Figure).

Another unique area comprises Mt. Petros and Mt. Berlebashka (aka Latundur). A series of alpine calcareous species occur here (Table 2), because limestone bedrock lies at a considerable altitude, which is unusual for the Ukrainian Carpathians. One of them is Genista tinctoria L. subsp. oligosperma (Andrae) Jáv., an East-South-Carpathian endemic, which does not occur anywhere else in the Ukrainian Carpathians. It is a mat-forming dwarf shrub, $10-15 \mathrm{~cm}$ high. The mats cover up to $300 \mathrm{~cm}^{2}$ and contain 20-40 flowering shoots. It is restricted to only two small localities (\#22, 28 ) in the subalpine zone. The area of each of them is ca. 150-200 $\mathrm{m}^{2}$. However, as follows from M. Deyl's (1940) monograph, the taxon inhabited a larger range of localities in the 1930s, which leads to an assumption that it has declined significantly by now.

The same habitats harbor Jovibarba globifera (L.) J. Parnell subsp. preissiana (Domin) Holub, a WestEast-Carpathian subendemic. Besides one locality on Mt. Berlebashka, its largest metapopulation inhabits the rocks on Mt. Petros and consists of several scattered subpopulations. They are replenished mainly by stolon growth. Numbers of rosettes of J. globifera subsp. preissiana ranges within 5-35 specimens in each subpopulation, while the percentage of flowering ramets may reach up to $22 \%$.

Similar metapopulation pattern and habitat requirements refer to other clonal high-mountain saxicolous calcareous species, Veronica fruticans Jacq. and Potentilla crantzii, which often occur together. Altitudinal range of Jovibarba globifera subsp. preissiana, $V$. fruticans, and P. crantzii on the rocks of the $\mathrm{S}$ and $\mathrm{E}$ aspects of Mt. Petros is $1530-1755 \mathrm{~m}$ a.s.l. (localities $18,19,21,22)$. Monitoring carried out in selected localities showed considerable increase in the numbers of flowering individuals of $J$. globifera subsp. preissiana and $V$. fruticans (2.6-4 and 2.8 times, respectively) in 14 years. This can be caused by the obvious decrease of grazing during that period. 
Table 2. Floristic composition in localities of rare plant species in the hotspots of Mts Petros-Berlebashka and the Bilyi Potik Valley (names of rare species in boldface)

\begin{tabular}{|c|c|c|c|c|c|c|c|c|c|c|c|c|c|}
\hline Number of locality & 18 & 19 & 20 & 21 & 22 & 23 & 24 & 25 & 26 & 27 & 28 & 29 & 30 \\
\hline Location & \multicolumn{7}{|c|}{ Mt. Petros } & \multicolumn{4}{|c|}{ Mt. Berlebashka } & \multicolumn{2}{|c|}{$\begin{array}{c}\text { Bilyi Potik } \\
\text { Valley }\end{array}$} \\
\hline Date of relevé (day, month, year) & 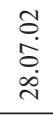 & 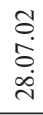 & 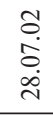 & $\begin{array}{l}0 \\
\hat{0} \\
\dot{0} \\
\text { o. }\end{array}$ & $\begin{array}{l}0 \\
\stackrel{1}{0} \\
\dot{0} \\
\dot{0}\end{array}$ & 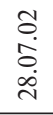 & 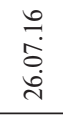 & 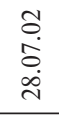 & $\begin{array}{l}\stackrel{0}{0} \\
\hat{\sigma} \\
\dot{0}\end{array}$ & 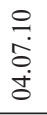 & $\begin{array}{l}0 \\
\stackrel{0}{0} \\
0 \\
\dot{i}\end{array}$ & $\begin{array}{l}0 \\
\stackrel{0}{0} \\
\stackrel{i}{0}\end{array}$ & $\begin{array}{l}\tilde{\delta} \\
\tilde{\sigma} \\
\dot{J} \\
\text { d. }\end{array}$ \\
\hline Area of relevé $\left(\mathrm{m}^{2}\right)$ & 25 & 5 & 100 & 50 & 100 & 25 & 10 & 100 & 100 & 25 & 100 & 25 & 25 \\
\hline Elevation (m a.s.l.) & $\tilde{n}$ & 吕 & $\stackrel{n}{E}$ & $\stackrel{n}{n}$ & $\stackrel{ }{\Xi}$ & గ్ర & $\frac{n}{6}$ & $\stackrel{n}{\stackrel{n}{2}}$ & 昂 & 은 & $\stackrel{n}{g}$ & \& & $\stackrel{n}{n}$ \\
\hline Exposition & $\mathrm{NE}$ & $\mathrm{E}$ & $\mathrm{N}$ & $\mathrm{S}$ & $\mathrm{S}$ & SW & SW & SE & $\mathrm{E}$ & $\mathrm{E}$ & $\mathrm{S}$ & $\mathrm{E}$ & $\mathrm{SE}$ \\
\hline Inclination $\left(^{\circ}\right)$ & 20 & 40 & 30 & 30 & 40 & 40 & 5 & 3 & 10 & 30 & 40 & 15 & 40 \\
\hline Vegetation cover $(\%)$ & 30 & 30 & 100 & 50 & 60 & 40 & 95 & 95 & 95 & 70 & 60 & 100 & 30 \\
\hline Achillea stricta & $\cdot$ & $\cdot$ & $\cdot$ & + & 1 & $\cdot$ & $\cdot$ & $\cdot$ & $\cdot$ & $\cdot$ & + & $\cdot$ & $\cdot$ \\
\hline Acinos alpinus & $\cdot$ & $\cdot$ & $\cdot$ & $\cdot$ & $\cdot$ & $\cdot$ & $\cdot$ & $\cdot$ & + & + & $\cdot$ & 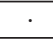 & $\cdot$ \\
\hline Aconitum anthora & . &. & . & . & $\cdot$ & + & . &. & $\cdot$ & $\cdot$ & $\cdot$ & . & $\cdot$ \\
\hline Anthoxanthum alpinum & $\cdot$ & $\cdot$ & $\cdot$ & $\cdot$ & 1 & $\cdot$ & $\cdot$ & 1 & . & $\cdot$ & 1 & $\cdot$ & $\cdot$ \\
\hline Aruncus sylvestris & $\cdot$ & $\cdot$ & $\cdot$ & $\cdot$ & $\cdot$ & $\cdot$ & $\cdot$ & $\cdot$ & $\cdot$ & $\cdot$ & $\cdot$ & + & 2 \\
\hline Asplenium trichomanes & $\cdot$ & $\cdot$ & $\cdot$ & $\cdot$ & $\cdot$ & $\cdot$ & $\cdot$ & $\cdot$ & $\cdot$ & $\cdot$ & $\cdot$ & + & + \\
\hline Asplenium viride & $\cdot$ & $\cdot$ & $\cdot$ & $\cdot$ & $\cdot$ & + & $\cdot$ & $\cdot$ & $\cdot$ & + & $\cdot$ & $\cdot$ & $\cdot$ \\
\hline Athyrium distentifolium & $\cdot$ & $\cdot$ & $\cdot$ & $\cdot$ & $\cdot$ & $\cdot$ & $\cdot$ &. & $\cdot$ & $\cdot$ & $\cdot$ & 2 & 2 \\
\hline Avenula pubescens & $\cdot$ & $\cdot$ & $\cdot$ & $\cdot$ & $\cdot$ & $\cdot$ & $\cdot$ & $\cdot$ & + & + & . & $\cdot$ & $\cdot$ \\
\hline Bellardiochloa violacea & $\cdot$ & $\cdot$ & $\cdot$ & $\cdot$ & 2 & $\cdot$ & $\cdot$ & $\cdot$ & $\cdot$ & $\cdot$ & $\cdot$ & $\cdot$ & $\cdot$ \\
\hline Calamagrostis arundinacea & 2 & 1 & $\cdot$ & $\cdot$ & 1 & $\cdot$ & $\cdot$ & $\cdot$ & $\cdot$ & $\cdot$ & $\cdot$ & . & $\cdot$ \\
\hline Calamagrostis villosa & $\cdot$ & $\cdot$ & 4 & $\cdot$ & $\cdot$ & $\cdot$ & $\cdot$ & $\cdot$ & $\cdot$ & $\cdot$ & 2 & $\cdot$ & $\cdot$ \\
\hline Campanula carpatica & $\cdot$ & 2 & $\cdot$ & $\cdot$ & $\cdot$ & + & $\cdot$ & $\cdot$ & $\cdot$ & $\cdot$ & $\cdot$ & 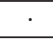 & 2 \\
\hline Campanula kladniana & $\cdot$ & $\cdot$ & $\cdot$ & + & $\cdot$ & $\cdot$ & $\cdot$ & $\cdot$ & $\cdot$ & $\cdot$ & 1 & $\cdot$ & $\cdot$ \\
\hline Campanula serrata & $\cdot$ & $\cdot$ & $\cdot$ & $\cdot$ & + & $\cdot$ & $\cdot$ & $\cdot$ & $\cdot$ & $\cdot$ & + & $\cdot$ & $\cdot$ \\
\hline Carduus kerneri & $\cdot$ & $\cdot$ & $\cdot$ & + & + & + & $\cdot$ & $\cdot$ & $\cdot$ & $\cdot$ & + & $\cdot$ & $\cdot$ \\
\hline Carex pauciflora & $\cdot$ & $\cdot$ & $\cdot$ & $\cdot$ & $\cdot$ & $\cdot$ & $\cdot$ & 3 & $\cdot$ & $\cdot$ & $\cdot$ & . & $\cdot$ \\
\hline Carex sempervirens & $\cdot$ & $\cdot$ & $\cdot$ & 1 & 1 & $\cdot$ & $\cdot$ & $\cdot$ & 2 & 2 & $\cdot$ & $\cdot$ & $\cdot$ \\
\hline Carlina acaulis & $\cdot$ & $\cdot$ & $\cdot$ & $\cdot$ & $\cdot$ & $\cdot$ & $\cdot$ & $\cdot$ & + & $\cdot$ & + & $\cdot$ & $\cdot$ \\
\hline Chrysosplenium alternifolium & $\cdot$ & $\cdot$ & $\cdot$ & $\cdot$ & $\cdot$ & $\cdot$ & $\cdot$ & $\cdot$ & $\cdot$ & $\cdot$ & $\cdot$ & + & $\cdot$ \\
\hline Cortusa matthioli & $\cdot$ & $\cdot$ & $\cdot$ & $\cdot$ & $\cdot$ & $\cdot$ & $\cdot$ & $\cdot$ & $\cdot$ & $\cdot$ & $\cdot$ & $\cdot$ & + \\
\hline Cotoneaster integerrimus & $\cdot$ & 1 & $\cdot$ & 1 & $\cdot$ & $\cdot$ & $\cdot$ & $\cdot$ & $\cdot$ & $\cdot$ & $\cdot$ & $\cdot$ & $\cdot$ \\
\hline Cruciata glabra & $\cdot$ & $\cdot$ & $\cdot$ & $\cdot$ & + & 1 & $\cdot$ & $\cdot$ & + & + & $\cdot$ & $\cdot$ & $\cdot$ \\
\hline Dactylorhiza cordigera & $\cdot$ & $\cdot$ & $\cdot$ & $\cdot$ & $\cdot$ & $\cdot$ & $\cdot$ & + & $\cdot$ & $\cdot$ & $\cdot$ & $\cdot$ & $\cdot$ \\
\hline Dactylorhiza fuchsii & $\cdot$ & $\cdot$ & $\cdot$ & $\cdot$ & + & $\cdot$ & $\cdot$ & $\cdot$ & $\cdot$ & $\cdot$ & $\cdot$ & $\cdot$ & $\cdot$ \\
\hline Daphne mesereum & $\cdot$ & $\cdot$ & $\cdot$ & $\cdot$ & $\cdot$ & + & $\cdot$ & $\cdot$ & + & + & $\cdot$ & $\cdot$ & $\cdot$ \\
\hline Deschampsia caespitosa & $\cdot$ & $\cdot$ & + & $\cdot$ & $\cdot$ & $\cdot$ & 2 & 2 & + & $\cdot$ & $\cdot$ & $\cdot$ & $\cdot$ \\
\hline Dianthus carthusianorum subsp. tenuifolius & + & $\cdot$ & $\cdot$ & + & $\cdot$ & $\cdot$ & $\cdot$ & $\cdot$ & $\cdot$ & $\cdot$ & + & $\cdot$ & $\cdot$ \\
\hline Euphorbia carpatica & $\cdot$ & $\cdot$ & $\cdot$ & $\cdot$ & $\cdot$ & $\cdot$ & $\cdot$ & $\cdot$ & + & + & $\cdot$ & $\cdot$ & $\cdot$ \\
\hline Fagus sylvatica & $\cdot$ & $\cdot$ & $\cdot$ & $\cdot$ & $\cdot$ & $\cdot$ & $\cdot$ & $\cdot$ & $\cdot$ & $\cdot$ & $\cdot$ & 3 & 2 \\
\hline Festuca airoides & 3 & 2 & $\cdot$ & 1 & 2 & $\cdot$ & $\cdot$ & $\cdot$ & $\cdot$ & 2 & 3 & $\cdot$ & $\cdot$ \\
\hline Festuca rubra & 2 & $\cdot$ & $\cdot$ & $\cdot$ & $\cdot$ & $\cdot$ & $\cdot$ & $\cdot$ & 2 & 3 & $\cdot$ & $\cdot$ & $\cdot$ \\
\hline Festuca saxatilis & $\cdot$ & $\cdot$ & $\cdot$ & $\cdot$ & 1 & $\cdot$ & $\cdot$ & $\cdot$ & $\cdot$ & $\cdot$ & $\cdot$ & $\cdot$ & $\cdot$ \\
\hline Fragaria vesca & $\cdot$ & $\cdot$ & $\cdot$ & $\cdot$ & $\cdot$ & $\cdot$ & $\cdot$ & $\cdot$ & + & + & $\cdot$ & $\cdot$ & $\cdot$ \\
\hline Genista tinctoria subsp. oligosperma & $\cdot$ & $\cdot$ & $\cdot$ & $\cdot$ & + & $\cdot$ & $\cdot$ & 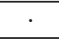 & $\cdot$ & $\cdot$ & 1 & $\cdot$ & $\cdot$ \\
\hline Gentiana acaulis & $\cdot$ & $\cdot$ & $\cdot$ & $\cdot$ & + & $\cdot$ & $\cdot$ & $\cdot$ & $\cdot$ & $\cdot$ & $\cdot$ & $\cdot$ & $\cdot$ \\
\hline Gentiana lutea & $\cdot$ & $\cdot$ & 1 & $\cdot$ & $\cdot$ & . &. & . & $\cdot$ & $\cdot$ & $\cdot$ & . & $\cdot$ \\
\hline Helianthemum nummularium subsp. grandiflorum & $\cdot$ & $\cdot$ & $\cdot$ & + & $\cdot$ & $\cdot$ & $\cdot$ & $\cdot$ & $\cdot$ & $\cdot$ & $\cdot$ & . & $\cdot$ \\
\hline Heracleum carpaticum & $\cdot$ & $\cdot$ & $\cdot$ & $\cdot$ & $\cdot$ & $\cdot$ & 1 & $\cdot$ & $\cdot$ & $\cdot$ & $\cdot$ & 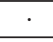 & $\cdot$ \\
\hline Hieracium umbellatum & $\cdot$ & $\cdot$ & $\cdot$ & $\cdot$ & $\cdot$ & $\cdot$ & $\cdot$ & $\cdot$ & $\cdot$ & + & + & $\cdot$ & $\cdot$ \\
\hline Hypochoeris uniflora & $\cdot$ & $\cdot$ & $\cdot$ & $\cdot$ & + & . & $\cdot$ & $\cdot$ & $\cdot$ & $\cdot$ & + & . & $\cdot$ \\
\hline Jovibarba globifera subsp. hirta & + & + & $\cdot$ & + & $\cdot$ & $\cdot$ & $\cdot$ & $\cdot$ & $\cdot$ & $\cdot$ & + & $\cdot$ & $\cdot$ \\
\hline Juniperus communis subsp. alpina & 2 & 2 & 1 & 2 & 1 & 2 & 2 & $\cdot$ & + & + & 1 & $\cdot$ & $\cdot$ \\
\hline Laserpitum alpinum & + & $\cdot$ & + & + & + & $\cdot$ & $\cdot$ & $\cdot$ & $\cdot$ & + & + & $\cdot$ & $\cdot$ \\
\hline
\end{tabular}




\begin{tabular}{|c|c|c|c|c|c|c|c|c|c|c|c|c|c|}
\hline Number of locality & 18 & 19 & 20 & 21 & 22 & 23 & 24 & 25 & 26 & 27 & 28 & 29 & 30 \\
\hline Linum extraaxillare & . & $\cdot$ & . & $\cdot$ & + & $\cdot$ & $\cdot$ & . & $\cdot$ & 1 & . & $\cdot$ & . \\
\hline Lotus corniculatus & $\cdot$ & $\cdot$ & $\cdot$ & + & & $\cdot$ & $\cdot$ & $\cdot$ & $\cdot$ & + & $\cdot$ & $\cdot$ & $\cdot$ \\
\hline Luzula luzuloides & . & . & 1 & + & 1 & . & 3 & . & . & $\cdot$ & 2 & . & . \\
\hline Parnassia palustris & . & . & . & . & . & . & $\cdot$ & + & . & + & $\cdot$ & . & . \\
\hline Phyllitis scolopendrium & $\cdot$ & $\cdot$ & $\cdot$ & $\cdot$ & $\cdot$ & $\cdot$ & $\cdot$ & $\cdot$ & $\cdot$ & $\cdot$ & $\cdot$ & 2 & $\cdot$ \\
\hline Phyteuma orbiculare & . & . & . & . & . & . & . & . & + & + & $\cdot$ & $\cdot$ & . \\
\hline Picea abies & 2 & 2 & + & . & $\cdot$ & 2 & 1 & . & $\cdot$ & . & $\cdot$ & $\cdot$ & . \\
\hline Poa nemoralis & 1 & 1 & . & . & 2 & 2 & $\cdot$ & . & $\cdot$ & 3 & . & $\cdot$ & . \\
\hline Polygala amara subsp. brachyptera & . & . & . & . & . & $\cdot$ & . & . & + & + & . & . & . \\
\hline Potentilla aurea & $\cdot$ & $\cdot$ & . & + & + & $\cdot$ & . & . & $\cdot$ & $\cdot$ & . & $\cdot$ & . \\
\hline Potentilla crantzii & 1 & + & . & 1 & + & + & $\cdot$ & . & $\cdot$ & + & . & . & . \\
\hline Prunella vulgaris & . & $\cdot$ & . & $\cdot$ & $\cdot$ & $\cdot$ & + & . & + & + & $\cdot$ & . & . \\
\hline Scabiosa lucida subsp. barbata & . & . & . & + & + & + & . & . & 1 & + & . & . & . \\
\hline Scopolia carniolica & . & $\cdot$ & . & $\cdot$ & $\cdot$ & $\cdot$ & . & $\cdot$ & $\cdot$ & $\cdot$ & $\cdot$ & 1 & + \\
\hline Scorzonera rosea & $\cdot$ & . & . & . & + & $\cdot$ & . & $\cdot$ & $\cdot$ & . & + & $\cdot$ & . \\
\hline Sedum telephium subsp. fabaria & 1 & . & . & $\cdot$ & $\cdot$ & 1 & . & . & . & $\cdot$ & $\cdot$ & . & . \\
\hline Silene dubia & $\cdot$ & + & . & + & + & $\cdot$ & . & $\cdot$ & $\cdot$ & + & + & $\cdot$ & $\cdot$ \\
\hline Stellaria holostea & . & $\cdot$ & $\cdot$ & $\cdot$ & + & . & $\cdot$ & $\cdot$ & $\cdot$ & $\cdot$ & + & $\cdot$ & $\cdot$ \\
\hline Stellaria nemorum & . & $\cdot$ & . & $\cdot$ & $\cdot$ & $\cdot$ & + & . & $\cdot$ & $\cdot$ & $\cdot$ & 1 & . \\
\hline Thymus alpestris & 2 & 2 & . & 1 & 2 & 1 & + & . & + & 2 & 1 & $\cdot$ & . \\
\hline Vaccinium myrtillus & 1 & 1 & 1 & 1 & 2 & 2 & . & . & $\cdot$ & $\cdot$ & 1 & $\cdot$ & . \\
\hline Vaccinium vitis-idaea & . & . & . & $\cdot$ & + & $\cdot$ & $\cdot$ & . & . & . & + & $\cdot$ & . \\
\hline Veronica fruticans & + & $\cdot$ & $\cdot$ & 1 & + & . & $\cdot$ & . & $\cdot$ & $\cdot$ & $\cdot$ & . & . \\
\hline
\end{tabular}

Other species occurring in a single locality (number in brackets) - Alchemilla monticola (26): +; Alnus viridis (22): +; Aposeris foetida (22): +; Asplenium septentrionale (19): +; Astrantia major (26): 1; Bellis perennis (26): +; Caltha laeta (25): +; Campanula abietina (24): +; C. glomerata (21): +; Cardaminopsis arenosa (23): +; Carex canescens (25): 2; C. echinata (25): 2; C. nigra (25): 1; Chrysosplenium alternifolium (29): +; Clematis alpina (30): 1; Corylus avellana (29): 3; Crepis conyzifolia (21): +; Cystopteris fragilis (19): +; Digitalis grandiflora (30): 1; Dryopteris carthusiana (23): 2; Epilobium collinum (23): +; E. roseum (29): +; Eriophorum polystachyon (25): 3; Galeobdolon luteum (29): 1; Geranium robertianum (29): +; Glechoma hederacea (29): 1; Hieracium auranthiacum (24): +; H. pilosella (27): +; H. richeri subsp. grisebachii (20): +; Hypericum maculatum (27): +; Impatiens noli-tangere (29): 2; Juncus conglomeratus (25): 1; J. trifidus (28): 2; Knautia dipsacifolia (26): 1; Lamium maculatum (29): +; Leucanthemum vulgare subsp. alpicola (21): +; Lilium martagon (21): +; Ligusticum mutellina (25): +; Lunaria rediviva (29): 1; Luzula sudetica (25): 1; L. sylvatica (24): +; Lysimachia nummularia (29): +; Melampyrum herbichii (21): +; Molinia caerulea (25): 2; Oxalis acetosella (24): +; Petasites albus (29): 2; Phleum alpinum (24): +; Polypodium vulgare (30): 2; Potentilla erecta (25): +; Ranunculus nemorosus (22): +; Rubus caesius (29): 2; R. idaeus (23): +; Salvia glutinosa (30): 2; Sambucus nigra (29): 2; Sedum atratum (18): +; Senecio papposus (22): +; Solidago virgaurea subsp. minuta (20): +; Spiraea chamaedryfolia (30): 1; Stachys sylvatica (29): +; Symphytum tuberosum (23): +; Tanacetum subcorymbosum subsp. clusii (21): +; Thesium alpinum (18): +; Trifolium repens (26): +; Urtica dioica (29): 2; Vaccinium gaultherioides (22): +; Valeriana tripteris (18): +; Veronica serpyllifolia (27): +; Plagiochila asplenioides (21): 1; Neckera crispa (30): 1; Sphagnum sp. (25): 4. Location of the sites: $18-47^{\circ} 57^{\prime} 30,5^{\prime \prime} \mathrm{N} 24^{\circ} 20^{\prime} 23,5^{\prime \prime} \mathrm{E} ; 19-47^{\circ} 57^{\prime} 33,5^{\prime \prime} \mathrm{N} 24^{\circ} 20^{\prime} 30,5^{\prime \prime} \mathrm{E} ; 20-47^{\circ} 57^{\prime} 46,5^{\prime \prime} \mathrm{N} 24^{\circ} 20^{\prime} 17,9^{\prime \prime} \mathrm{E} ; 21-47^{\circ} 57^{\prime} 42,4^{\prime \prime} \mathrm{N}$ $24^{\circ} 20^{\prime} 11,8^{\prime \prime} \mathrm{E} ; 22-47^{\circ} 57^{\prime} 40,0^{\prime \prime} \mathrm{N} 24^{\circ} 20^{\prime} 10,9^{\prime \prime} \mathrm{E} ; 23-47^{\circ} 57^{\prime} 35,9^{\prime \prime} \mathrm{N} 24^{\circ} 20^{\prime} 05,6^{\prime \prime} \mathrm{E} ; 24-47^{\circ} 57^{\prime} 34,3^{\prime \prime} \mathrm{N} 24^{\circ} 20^{\prime} 04,5^{\prime \prime} \mathrm{E}$; $25-47^{\circ} 57^{\prime} 16,9^{\prime \prime} \mathrm{N} 24^{\circ} 19^{\prime} 38,8^{\prime \prime} \mathrm{E} ; 26-47^{\circ} 56^{\prime} 51,9^{\prime \prime} \mathrm{N} 24^{\circ} 19^{\prime} 04,9^{\prime \prime} \mathrm{E} ; 27-47^{\circ} 57^{\prime} 54,3^{\prime \prime} \mathrm{N} 24^{\circ} 19^{\prime} 01,7^{\prime \prime} \mathrm{E} ; 28-47^{\circ} 57^{\prime} 06,3^{\prime \prime} \mathrm{N}$ $24^{\circ} 18^{\prime} 58,3^{\prime \prime} \mathrm{E} ; 29-47^{\circ} 56^{\prime} 08,9^{\prime \prime} \mathrm{N} 24^{\circ} 15^{\prime} 26,1^{\prime \prime} \mathrm{E} ; 30-47^{\circ} 55^{\prime} 49,4^{\prime \prime} \mathrm{N} 24^{\circ} 15^{\prime} 22,0^{\prime \prime} \mathrm{E}$. 
We also discovered other positive examples of some rare species dynamics, namely establishment of their new populations on limestone bedrock on the $\mathrm{S}$ slope of Mt. Berlebashka. Thus, in 2010 we found a few individuals of Potentilla crantzii on anthropogenic outcrops of limestone that had appeared on the sideslope of a recently constructed road (locality 27). The species was missing there in 2002, whereas its quite viable population has developed at the site by 2016 . Most probably, $P$. crantzii has dispersed from its large «mainland» population on Mt. Petros situated ca. $2 \mathrm{~km}$ eastwards.

A newly established population of Acinos alpinus (L.) Moench (=A. baumgartenii (Simk.) Klokov) was first found near that site on a pasture (locality 26) in 2010. It occupied about $500 \mathrm{~m}^{2}$ area then, but has expanded to ca. $2000 \mathrm{~m}^{2}$ by 2016 . However, the average density of flowering individuals of $A$. alpinus has not changed significantly remaining within $1.1-1.3$ specimens per $\mathrm{m}^{2}$.

Quite opposite dynamics was revealed for Saxifraga adscendens L., an arctic-alpine species that occurred in the same locality in 2001, but was not found there in the later surveys.

The same refers to Koeleria macrantha (Ledeb.) Schult. subsp. transsilvanica (Schur) A. Nyár., an EastSouth-Carpathian endemic, which was reported from the S slope of Mt. Petros by M. Deyl (1940), but was never confirmed later. That author also indicated the altitude of the locality and published its phytosociological relevé, with a very peculiar floristic composition, which enabled to locate the site correctly (locality 22) and to make sure that $K$. macrantha subsp. transsilvanica has become extinct in its only known Ukrainian habitat. Its extinction could be caused by considerable decrease of grazing and consequent overgrowth of its habitat with more competitive graminoids or shrubs.

Nevertheless, Festuca saxatilis Schur, another EastSouth-Carpathian endemic, has persisted in the same locality, which is the only one we managed to find in the region. This saxicolous species forms dense tufts and occurs within about $25 \mathrm{~m}^{2}$. That locality is also inhabited by a large population of Bellardiochloa violacea (Bellardi) Chiov.

A limestone rock in the spruce forest at the SW foothill of Mt. Petros provides a habitat for some rare calcareous species, e.g. Aconitum anthora L. (= A. jacquinii Rchb.), Campanula carpatica, Symphytum tuberosum L. (locality $23)$. The first of them is very rare in the region and its population numbered only 14 flowering individuals in 2016, whereas $C$. carpatica is more common on the rocks in the forest zone.

On the same limestone bedrock nearby the abovementioned habitat we found a small population of Heracleum carpaticum Porcius, an East-SouthCarpathian endemic (locality 24). It occupied only ca. $10 \mathrm{~m}^{2}$ in 2016 and contained 6 flowering and 19 vegetative individuals. This aclonal species is replenished only due to seed reproduction. The habitat conditions are rather unusual for that alpine species, which is confined to a forest glade there at a comparatively low elevation.

Another East-South-Carpathian endemic, Silene nutans L. subsp. dubia (Herbich) Zapał., is rather common on Mt. Petros and Mt. Berlebashka and forms a large metapopulation. Helianthemum nummularium (L.) Mill. subsp. grandiflorum (Scop.) Schinz \& Thell., a high-mountain Central European subspecies, was found only in one locality (\#21) on Mt. Petros.

A large population of Gentiana lutea stretches along steep hollows on the N and NE slopes of Mt. Petros near its summit (locality 20). The population is confined to $1660-1770 \mathrm{~m}$ a.s.1., occupies about $5000 \mathrm{~m}^{2}$ and numbers $1.5-2.0$ thousand flowering individuals.

Polygala amara L. subsp. brachyptera (Chodat) Hayek, a narrow-range montane taxon, occurs in some calciumrich saxicolous or grassland sites (localities 4, 26, 27).

The last noteworthy "hotspot" of the rare species is the Bilyi Potik Valley, which is remarkable for numerous cliffs of marbelized limestones situated in the beech forest zone at much lower elevations in comparison with the listed above localities. Campanula carpatica is very abundant on such cliffs and smaller outcrops and forms lax mats, with up to 3 flowering shoots per $100 \mathrm{~cm}^{2}$.

Cortusa matthioli L., a saxicolous chasmophite, inhabits shady rock fissures. Its largest population (locality 30) contains over 100 flowering individuals. The species number is limited by the availability of suitable microhabitats and low seed productivity (Kobiv, 1999).

Some publications (Shushman, 2008) and herbarium data indicate that a very rare saxicolous species, Sempervivum marmoreum L., also occurs on the cliffs near the Bilyi Potik stream, but we failed to find it.

Tall-forb communities beneath the rocks harbor Phyllitis scolopendrium (L.) Newman and Scopolia carniolica Jacq. Their average density is 1.2 and 0.4 individuals per $1 \mathrm{~m}^{2}$, respectively (locality 29).

Calcicolous flora of these habitats is typical of the belt of Pieniny and Marmarosh limestone cliffs, which stretches along the border between the Inner and Outer Carpathians. 
Table 3. Soil parameters in selected localities of rare plant species in the Marmarosh Mts

\begin{tabular}{|c|c|c|c|c|c|c|c|c|c|c|c|c|}
\hline Number of locality & 2 & 3 & 5 & 8 & 9 & 14 & 15 & 17 & 21 & 22 & 28 & 30 \\
\hline $\mathrm{pH}\left(\mathrm{H}_{2} \mathrm{O}\right)$ & 5.86 & 6.78 & 6.56 & 3.75 & 4.15 & 3.91 & 4.76 & 5.21 & 3.86 & 4.40 & 6.19 & 7.05 \\
\hline Content of $\mathrm{Ca}$ (mg-eq./100 g) & 34.83 & 12.82 & 24.40 & 1.52 & 1.95 & 9.80 & 12.42 & 13.60 & 9.60 & 10.81 & 18.40 & 36.24 \\
\hline Content of Mg (mg-eq./100 g) & 6.02 & 3.22 & 4.80 & 1.50 & 1.62 & 8.15 & 4.01 & 11.21 & 8.00 & 4.82 & 6.40 & 17.31 \\
\hline
\end{tabular}

Because of steep topography, wetlands are infrequent in the Marmarosh Mts. The largest peat bogs that cover 100-750 $\mathrm{m}^{2}$ (localities 6, 25) are inhabited by Carex limosa L. and C. pauciflora Lightf.

In many of his relevés M. Deyl (1940) mentioned Avenula pubescens (Huds.) Dumort. subsp. laevigata (Schur) Holub, which could imply that this narrowrange montane plant is rather common in the Marmarosh Mts. Apparently, that results from misidentification, because thorough examination of the specimens, which we collected in the region (including the localities stated by that author) and their comparison with the type material (Nachychko et al., 2017) proved that they belong to $A$. pubescens subsp. pubescens, and the former taxon hardly occurs in Ukraine.

Soil analysis in the selected localities of rare species (Table 3) showed the highest $\mathrm{pH}$ values and content of calcium on the limestone cliffs in the Bilyi Potik Valley. Rocky habitats of Mt. Nenieska are also rich in calcium. By contrast, most acidic and calcium-poor soil is in the snowbed locality in the glacial cirque of Mt. Pip Ivan. Almost equal content of calcium and magnesium was noted in some localities on crystalline bedrock on Mt. Pip Ivan and Mt. Petros, whereas the former element prevails significantly in most soils all over the Ukrainian Carpathians. Apparently, presence of markedly basiphilous species in some of these localities is considerably contributed by magnesium.

\section{Conclusion}

In biogeographical terms, the Marmarosh Mountains are the area of remarkable biodiversity, where many rare plants occur. Some of them, namely Anthemis cretica subsp. pyrethriformis, Genista tinctoria subsp. oligosperma, Potentilla crantzii and Veronica bellidioides, do not occur elsewhere in Ukraine, because edges of their geographical ranges slightly protrude into the Ukrainian territory only in that area, while they are much more abundant in the Romanian Carpathians. That floristic specificity is caused mostly by the geological structure of the region, which differs from the rest of the Ukrainian Carpathians. Other rare range-edge species that in addition to the Marmarosh Mts have a very limited distribution in some other frontier regions of the Ukrainian Carpathians are: Festuca saxatilis, Jovibarba globifera subsp. preissiana, Sempervivum marmoreum, Veronica fruticans. Some of the listed species, for instance, Veronica bellidioides, are critically endangered, while Koeleria macrantha subsp. transsilvanica, has aready become extinct in the Marmarosh Mts. The localities of these taxa in the Marmarosh Mts are confined to the northeastern limit of their distribution in Central Europe and their extinction causes contraction of the species' geographical ranges and possible irreversible loss of biodiversity, because some peripheral populations have a unique gene pool (Lesica, Allendorf, 1995). As follows from the above results, many rare species of the Marmarosh Mts are narrow-range and the mentioned facts of decline or extinction are most worrying for the endemics (Genista tinctoria subsp. oligosperma, Koeleria macrantha subsp. transsilvanica). Decline of coldadapted alpine species (Cerastium cerastoides, Luzula spicata, Primula minima, Saxifraga carpatica, Veronica bellidioides) has taken place mainly at the lower limit of their ecological range and can be explained by climate change.

Another factor that affects rare species is considerable decrease of grazing in the region, which has been observed lately. Along with climate change it triggers natural succession of vegetation in the habitats of some rare species and their replacement by highly competitive tussock graminoids or shrubs. For that reason, mild grazing regime that still remains in some open habitats of saxicolous or chionophylous species, e.g. on Mts Nenieska, Pip Ivan, Petros, Berlebashka is favorable for their persistence, because it mitigates adverse changes in these localities. This refers mostly to low-statured poorly competitive species (e.g. Acinos alpinus, Cerastium cerastoides, Saxifraga adscendens, S. carpatica, Veronica bellidioides), which need gaps in vegetation for their recruitment (Virtanen et al., 1997). The mitigating effect of grazing against warminginduced decline of alpine species has been proved experimentally (Klein et al., 2004).

On the other hand, another group of species has benefited from the decrease of grazing. They demonstrate their highest viability in undisturbed conditions. This 
concerns primarily tall forbs (Heracleum sphondylium subsp. transsilvanicum, Gentiana punctata), but also refers to some rare saxicolous components of grassland or dwarf-shrub communities (Jovibarba globifera subsp. preissiana, Veronica fruticans).

Thus, various groups of rare species have different needs for their effective conservation, which should be carefully considered in implementation of land-use measures in their habitats. Supposedly, their persistence in open saxicolous and chionophilous localities could be best provided by maintenance of mild grazing regime to prevent overgrowth of their habitats with highly competitive species.

By contrast with the above-mentioned extinction events, we have revealed a few facts of colonization of new habitats by some rare species (Acinos alpinus, Potentilla crantzii), which happened recently in anthropogenically disturbed localities.

Thus, populations of many rare species in the Marmarosh Mts undergo continuing transformations responding to changes in climatic factors and varying anthropogenic pressure or resulting from succession of vegetation in their habitats.

Almost all species mentioned in this article are listed in the Red Data Book of Ukraine (Chervona knyha..., 2009). Our survey proved that some studied taxa should be included into its next edition, namely, Koeleria macrantha subsp. transsilvanica and Luzula spicata, both as extinct (EX); however, a few historical localities of the latter species in the Chornohora still need to be thoroughly checked for its presence.

Though Potentilla crantzii is rare and confined to a very small area in Ukraine, it is not prone to decline and therefore does not require any conservation status.

\section{Acknowledgements}

We are very grateful to I. Rabyk, Institute of Ecology of the Carpathians, NASU for determining the bryophytes.

\section{REFERENCES}

Arinushkina E.V. Rukovodstvo po khimicheskomu analizu pochv, Moscow: Izd-vo Mosk. Univ., 1970, 487 pp. [Аринушкина Е.В. Руководство по химическому анализу почв, М.: Изд-во Моск. ун-та, 1970, 487 с.].

Braun-Blanquet J. Pflanzensoziologie, Grundzüge der Vegetationskunde. Ed. 3, Wien; New York: Springer, 1964, $865 \mathrm{pp}$.

Chervona knyha Ukrainy. Roslynnyi svit (Red Data Book of Ukraine. Plant Kingdom). Ed. Ya.P. Didukh, Kyiv: Globalkonsaltyng, 2009, 912 pр. [Червона книга України. Рослинний світ. Ред. Я.П. Дідух, Київ: Глобалконсалтинг, 2009, 912 с.].
Chopyk V.I. Vysokohirna flora Ukrainskykh Karpat, Kyiv: Naukova Dumka, 1976, 270 pp. [Чопик B.I. Високогірна флора Українських Карпат, Київ: Наук. думка, 1976, 270 c.].

Deyl M. Plants, soil and climate of Pop Ivan. Synecological study from Carpathian Ukraine. Opera Bot. Čechica, 1940, 2: 1-290.

Ellenberg H., Weber H.E., Düll R., Wirth V., Werner W., Paulissen D. Zeigerwerte von Pflanzen in Mitteleuropa. 2 Auflage. Scripta Geobot., 1992, 18: 1-258.

Elzinga C.L., Salzer D.W., Willoughby J.W., Gibbs J.P. Monitoring plant and animal populations: A handbook for field biologists, Chichester: John Wiley \& Sons, 2009, 368 pp.

Flora Europaea, Cambridge: Cambridge Univ. Press, 19641980, vols 1-5.

Flora URSR (Flora RSS Ucr.), Kyiv: Vyd-vo Acad. Sci. URSR, 1950-1965, vols 3-12. [Флора УРСР, Київ: Вид-во АН УРСР, 1950-1965, т. 3-12].

Frei E., Bodin J., Walther G.R. Plant species' range shifts in mountainous areas - all uphill from here? Bot. Helv., 2010, 120(2): 117-128. doi:10.1007/s00035-010-0076-y.

Harper J.L. Population biology of plants, New York; London; San Francisco: Acad. Press, 1977, 892 pp.

Hill M.O., Bell N., Bruggeman-Nannenga M.A., Brugués M., Cano M.J., Enroth M.J., Flatberg K.I., Frahm J.-P., Gallego M.T., Garilleti R., Guerra J., Hedenäs L., Holyoak D.T., Hyvönen J., Ignatov M.S., Lara F., Mazimpaka V., Muñoz J., Söderström L. An annotated checklist of the mosses of Europe and Macaronesia. J. Bryol., 2006, 28(3): 198-267. doi:10.1179/174328206X119998.

Klášterský I. Ad floram Carpatorossicam additamenta critica I. Preslia, 1929, 8: 9-32; - II, 1930, 9: 5-21; III, 1931, 10: 76-87.

Klein J.A., Harte J., Zhao X.-Q. Experimental warming causes large and rapid species loss, dampened by simulated grazing, on the Tibetan Plateau. Ecol. Letters, 2004, 7: 1170-1179. doi:10.1111/j.1461-0248.2004.00677.x.

Kliment J., Turis P., Janišova M. Taxa of vascular plants endemic to the Carpathian Mts. Preslia, 2016, 88: 19-76.

Kobiv Y. Cortusa matthioli L. (Primulaceae) in the Chornohora Mts (Ukrainian Carpathians): distribution, ecology, taxonomy and conservation. Fragm. Florist. Geobot., 1999, 44(2): 355-574.

Kobiv Y.Y. Veronica bellidioides. In: Chervona knyha Ukrainy. Roslynnyi svit (Red Data Book of Ukraine. Plant Kingdom). Ed. Ya.P. Didukh, Kyiv: Globalkonsaltyng, 2009, p. 617. [Кобів Ю.Й. Veronica bellidioides L. В кн.: Червона книга України. Рослинний світ. Ред. Я.П. Дідух, Київ: Глобалконсалтинг, 2009, с. 617].

Kobiv Y.Y. Ukr. Bot. J., 2010, 67(3): 355-372. [Кобів Ю.Й. Екологічні особливості оселищ рідкісних видів рослин Українських Карпат. Укр. бот. жжурн., 2010, 67(3): 355-372].

Kobiv Y.Y. Ukr. Bot. J., 2012, 69(2): 178-189. [Кобів Ю.Й. Роль придатних мікрооселищ у самовідновленні популяцій рідкісних видів рослин Українських Карпат. Укр. бот. журн., 2012, 69(2): 178-189]. 
Kondracki J. Karpaty, Warszawa: Wydawnictwo Szkolne i Pedagogiczne, 1989, $262 \mathrm{pp}$.

Krist V. O některých nových nebo kritických rostlinach na Slovensku a Podkarpatské Rusi. Spisy Př́r. Fak. Masarykovy Univ., 1935, 199: 1-16.

Kruhlov I., Ukr. Geogr. J., 2008, 3: 59-68. [Круглов I. Делімітація, метризація та класифікація морфогенних екорегіонів Українських Карпат. Укр. геогр. журн., 2008, 3: 59-68].

Lesica P., Allendorf F.W. When are peripheral populations valuable for conservation? Conserv. Biol., 1995, 9(4): 753-760.

Malynovski K.A. Roslynnist vysokohirya Ukrainskykh Karpat, Kyiv: Naukova Dumka, 1980, 280 рр. [Малиновський К.А. Рослинність високогір'я Українських Kapnam, Київ: Наук. думка, 1980, 280 с.].

Malynovski K., Tsaryk Y., Kyyak V., Nesteruk Y. Ridkisni, endemichni, reliktovi ta pohranychno-arealni vydy roslyn Ukrainskykh Karpat, Lviv: Liga-Press, 2002, 78 pp. [Maлиновський К., Царик Й., Кияк В., Нестерук Ю. Рідкісні, ендемічні, реліктові та погранично-ареальні види рослин Українських Карпат, Львів: Ліга-Прес, 2002, 78 c.].

Micu D. Snow pack in the Romanian Carpathians under changing climatic conditions. Meteorol. Atmos. Phys., 2009, 105: 1-16. doi:10.1007/s00703-009-0035-6.

Nachychko V.O., Kobiv Y.Y., Sosnovsky Y.V., Helesh M.B., Prokopiv A.I. Typification of the name Avena laevigata, the basionym of Avenula pubescens subsp. laevigata (Poaceae). Phytotaxa, 2017, 299(2): 285-288. doi:10.11646/ phytotaxa.299.2.14

Pauli H., Gottfried M., Reiter K., Klettner C., Grabherr G. Signals of range expansions and contractions of vascular plants in the high Alps: observations (19942004) at the GLORIA master site Schrankogel, Tyrol, Austria. Global Change Biology, 2007, 13: 147-156. doi:10.1111/j.1365-2486.2006.01282.x.
Shushman V.S. In: Fitoriznomanittya Karpat: suchasnyi stan, okhorona ta vidtvorennya: mat. Mizhnar. nauk. konf. (Phytodiversity of the Carpathians: modern status, conservation and restoration: Proc. Int. Sci. Conf.), Uzhhorod: Lira, 2008, pp. 177-180. [Шушман В.С. Ботанічні нотатки про цікаві знахідки. В зб.: Фіторізноманіття Карпат: сучасний стан, охорона та відтворення: мат. Міжнар. наук. конф., Ужгород: Ліра, 2008, с. 177-180].

Szczęśniak E., Krukowski M., Malicki M. Pojawy i stopień zagrożenia Veronica bellidioides (Scrophulariaceae) w Polsce. Fragm. Flor. Geobot. Polonica, 2011, 18(1): 3-9.

Virtanen R., Henttonen H., Laine K. Lemming grazing and structure of a snowbed plant community: a long-term experiment at Kilpisjärvi, Finnish Lapland. Oikos, 1997, 79(1): 155-166. doi:10.2307/3546100.

Zapałowicz H. Roślinna szata Gór Pokucko-Marmaroskich. Spraw. Komis. Fizjogr., 1889, 24: 1-390.

Zarzycki K., Trzcińska-Tacik H., Różański W., Szelag Z., Wołek J., Korzeniak U. Ecological indicator values of vascular plants of Poland, Kraków: W. Szafer Inst. Bot., Polish Acad. Sci., 2002, 183 pp.

Ziman S.N., Bulakh E.V., Hamor A.F. "Hot spots" within the high-mountain floras as the sectors of the integrity of the rare species (on the model of the Ukrainian Carpathians). In: Plant, fungal and habitat diversity investigation and conservation: Proc. IV Balkan Bot. Congr., Sofia: Inst. Bot., Bulgar. Acad. Sci., 2009, pp. 555-561.

Recommended for publication by Submitted 16.02.2017 I.A. Korotchenko 
Кобів Ю. ${ }^{1,2}$, Прокопів А. ${ }^{2}$, Начичко В. ${ }^{2}$, Борсукевич Л. ${ }^{2}$, Гелеш M. ${ }^{2}$ Поширення і стан популяцій рідкісних видів рослин у Мармароських горах (Українські Карпати). Укр. бот. журн., 2017, 74(2): 163-176.

${ }^{1}$ Інститут екології Карпат НАН України вул. Козельницька, 4, Львів 790026, Україна

${ }^{2}$ Ботанічний сад Львівського національного університету ім. Івана Франка вул. Черемшини, 44, Львів 790014, Україна

У статті подано результати обстежень оселищ рідкісних видів рослин у Мармароських горах (Українські Карпати) та описано їхнє точне розташування й популяційні параметри. У результаті аналізу попередніх літературних і гербарних матеріалів встановлено деякі просторовочасові закономірності їхнього поширення, зокрема особливості оселищ і динаміки популяцій, що уможливило визначення їхнього природоохоронного статусу. Виявлено численні факти регресування чи зникнення, а також приклади позитивної динаміки популяцій досліджених видів. Холодостійкі види (Cerastium cerastoides, Primula minima, Veronica bellidioides) виявилися вразливими до кліматичних змін, зокрема на нижній межі їхнього поширення. Іншим фактором впливу є послаблення випасання, що спричинило сукцесію рослинності i, як наслідок, витіснення деяких низькоконкурентних рідкісних рослин (Genista tinctoria subsp. oligosperma, Koeleria macrantha subsp. transsilvanica) чагарничками або дернинними злаками. Натомість, інша група рідкісних видів (Heracleum sphondylium subsp. transsilvanicum, Gentiana punctata, Jovibarba globifera subsp. preissiana, Veronica fruticans) позитивно реагує на зменшення антропогенного впливу.

Ключові слова: зміни клімату, вимирання, динаміка популяцій, локалітет, Карпати
Кобив Ю. ${ }^{1,2}$, Прокопив А. ${ }^{2}$, Начычко В. ${ }^{2}$, Борсукевич Л. ${ }^{2}$, Гелеш М. ${ }^{2}$ Распространение и состояние популяций редких видов растений в Мармарошских горах (Украинские Карпаты). Укр. бот. журн., 2017, 74(2): 163-176.

${ }^{1}$ Институт экологии Карпат НАН Украины ул. Козельницкая, 4, Львов 79026, Украина

${ }^{2}$ Ботанический сад Львовского национального университета им. Ивана Франко ул. Черемшины, 44, Львов 79014, Украина

Представлены результаты обследования местопроизрастаний редких видов растений в Мармарошских горах (Украинские Карпаты) и описаны их точное расположение и популяционные параметры. В результате анализа предыдущих литературных и гербарных данных установлены некоторые пространственно-временные закономерности их распространения, в частности особенности местообитаний, тенденции динамики популяций, что дало возможность определить их природоохранный статус. Выявлены многочисленные факты их регрессирования или исчезновения, а также примеры положительной динамики популяций исследованных видов. Холодоустойчивые виды (Cerastium cerastoides, Primula minima, Veronica bellidioides) оказались уязвимыми к климатическим изменениям, в частности на нижней границе их распространения. Еще одним влияющим фактором является ослабление выпаса, вызвавшее сукцессию растительности и последующее вытеснение некоторых низкоконкурентных редких растений (Genista tinctoria subsp. oligosperma, Koeleria macrantha subsp. transsilvanica) кустарничками или дерновинными злаками. Однако, другая группа редких видов (Heracleum sphondylium subsp. transsilvanicum, Gentiana punctata, Jovibarba globifera subsp. preissiana, Veronica fruticans) положительно реагирует на уменьшение антропогенного влияния.

Ключевые слова: изменения климата, вымирание, динамика популяций, локалитет, Карпаты 